\title{
First-principles predictions of ruthenium-phosphorus and ruthenium-boron glassy structures and chemical vapor deposition of thin amorphous ruthenium-boron alloy films
}

\author{
Daniel E. Bost, Hyun-Woo Kimª ${ }^{\text {a }}$ Chia-Yun Chou, Gyeong S. Hwang, John G. Ekerdt ${ }^{\S}$
}

\begin{abstract}
:
First-principles density-functional calculations are presented revealing that $\mathrm{Ru}(\mathrm{P})$ and $\mathrm{Ru}(\mathrm{B})$ alloys with moderate $\mathrm{P}$ or $\mathrm{B}$ content can result in a glassy structure exhibiting strong chemical short-to-medium range order. Amorphous phases are predicted to be energetically more favorable than the crystalline counterparts for the $\mathrm{Ru}(\mathrm{P})$ and $\mathrm{Ru}(\mathrm{B})$ alloys above 20 at.\% $\mathrm{P}$ and $\sim 10$ at.\% $\mathrm{B}$. The relative stability of amorphous and crystalline $\mathrm{Ru}(\mathrm{B})$ alloys is examined along with local atomic ordering in the amorphous alloys. The growth of ultrathin $(3 \mathrm{~nm})$ amorphous $\mathrm{Ru}(\mathrm{B})$ alloy films of varying $\mathrm{B}$ concentration via chemical vapor deposition is explored using $\mathrm{Ru}_{3}(\mathrm{CO})_{12}$ and $\mathrm{B}_{2} \mathrm{H}_{6}$ as the $\mathrm{Ru}$ and $\mathrm{B}$ sources, respectively. Experiments reveal the films grown at $250^{\circ} \mathrm{C}$ are amorphous at B contents in excess of 15 at. $\%$ and polycrystalline below 10 at.\% B, consistent with first-principles predictions. Amorphous $\mathrm{Ru}(\mathrm{B})$ films remain amorphous following annealing at $450{ }^{\circ} \mathrm{C}$ and become polycrystalline at $500{ }^{\circ} \mathrm{C}$. Film resistivity ranged from 40 to $120 \mu \Omega-\mathrm{cm}$ and was independent of B loading. Electric field stress tests to failure for $\mathrm{Cu} / 3-\mathrm{nm} \mathrm{Ru}(\mathrm{B}) / \mathrm{SiO}_{2} / \mathrm{Si}$ stacks are used to indicate suitability of $\mathrm{Ru}(\mathrm{B})$ as a copper diffusion barrier layer.
\end{abstract}


Keywords: Amorphous film, Ruthenium alloy, Copper diffusion barrier, Boron, $\mathrm{Ru}(\mathrm{B})$ 


\section{1 - Introduction:}

As the characteristic dimensions of microelectronic devices shrinks and RC delay becomes a more significant factor in device design, interest in extremely thin, platable, conformal diffusion barriers for copper interconnects has grown [1], with industry predictions indicating the necessity of a conformal barrier layer $1.9 \mathrm{~nm}$ thick for the $16-\mathrm{nm}$ technology node [2]. A two-layer barrier of $\mathrm{Ta}$ and $\mathrm{TaN}$ is usually the base-line performance against which other materials are compared due to thermodynamic stability with respect to $\mathrm{Cu}$ and compatibility with many low-k materials and $\mathrm{SiO}_{2}[3,4]$. As devices continue to shrink, however, the two-layer method has become increasingly impractical, most noticeably due to the challenges of depositing the $\mathrm{Cu}$ seed layer and the increasing role of $\mathrm{Cu}$ diffusion along crystalline grain boundaries for low $(<10 \mathrm{~nm})$ barrier thicknesses $[5,6,7]$.

Several responses to this have been proposed and tested. Atomic layer deposition (ALD) and plasma-assisted deposition have been used to grow single-crystal films with few or no grain boundaries using known materials such as $\mathrm{TaN}_{\mathrm{x}}[8]$. Other materials such as nitrogen have been used to stuff the grain boundaries, either by saturating the material during growth $[5,9]$ or depositing a secondary layer of material that diffuses and fills the grain boundaries after growth [10]. A variety of metal alloys such as $\operatorname{Ru}_{\mathrm{x}} \mathrm{Ta}_{\mathrm{y}}$ [11], $\mathrm{Ti}(\mathrm{N})$ [12], W-doped indium oxides [13], Co-W alloys [5], $\mathrm{Ru}(\mathrm{P})$ [14], and $\mathrm{VO}_{\mathrm{x}}$ and self-forming $\mathrm{V}-\mathrm{Cu}$ alloys [15] have also been examined for barrier properties at low thickness. Some three-element alloys and non-metallic components have also shown promising results, such as TaSiC [16] and WGeN [17], and graphene [2]. Herein, we examine the elimination of grain boundaries by the addition of an alloying element during chemical vapor deposition (CVD) to cause the potential barrier material to grow in an amorphous rather than crystalline phase. 
Ruthenium grown by CVD or ALD has arisen as a potential single-layer alternative to $\mathrm{Ta} / \mathrm{TaN}$ stacks $[6,11,18,19,20,21]$. Previous studies of Ru in the 10-nm thickness range have shown the addition of $\mathrm{P}$ causes the $\mathrm{Ru}(\mathrm{P})$ alloy to grow as an amorphous film $[6,20]$. Ruthenium films containing $\sim 17$ at.\% of $\mathrm{P}$ remain amorphous upon annealing to $360{ }^{\circ} \mathrm{C}[14,22]$. Further, 5 and 3-nm-thick amorphous $\mathrm{Ru}(\mathrm{P})$ films function as $\mathrm{Cu}$ diffusion barriers [7, 14,]. $A b$ initio molecular dynamics calculations [6] showed that the $\mathrm{Ru}(\mathrm{P})$ alloy with 20 at.\% $\mathrm{P}$ can result in an amorphous structure exhibiting the topological and strong short-range order.

Generally, the atomic size ratio and negative formation energy are considered to be the main parameters to promote the properties of amorphous alloys. To gain quantitative understanding of the effect of the atomic size ratio $(\lambda)$ and alloying elements on amorphous alloy formation ability we experimentally and computationally examine boron (B), which is different in atomic size and chemistry with $\mathrm{P}$, in creating an amorphous $\mathrm{Ru}(\mathrm{B})(a-\mathrm{Ru}(\mathrm{B}))$ alloy as an alternative to amorphous $\mathrm{Ru}(\mathrm{P})(a-\mathrm{Ru}(\mathrm{P}))$ alloys.

We use first principles density-functional theory calculations to predict the three dimensional (3D) amorphous alloy structures and bonding properties of $\mathrm{Ru}(\mathrm{P})$ and $\mathrm{Ru}(\mathrm{B})$ alloys. Analyzing the details of the structure changes in the different alloy systems reveals icosahedra dominate the medium range ordering in both systems, while the short range ordering is primarily governed by the atomic size ratio. Twenty at.\% $\mathrm{P}$ was found to favor $a-\mathrm{Ru}(\mathrm{P})[6,14]$ and results reported herein indicate an $a$ - $\mathrm{Ru}(\mathrm{B})$ film is favored above 10 at.\% B. This study also reports the use of $\mathrm{CVD}$ to produce $a-\mathrm{Ru}(\mathrm{B})$ thin films at $\mathrm{B}$ content above 10 at. $\%$ using $\mathrm{Ru}_{3}(\mathrm{CO})_{12}$ as the $\mathrm{Ru}$ source and $\mathrm{B}_{2} \mathrm{H}_{6}$ as the $\mathrm{B}$ source. Diborane is selected because it decomposes completely on $\mathrm{Ru}(0001)$ between 230 and $400 \mathrm{~K}$, depending on coverage [23], making it amenable to use at typical film growth temperatures. 


\section{2 - Equipment and procedures}

The equipment and procedures used to produce and analyze $\mathrm{Ru}(\mathrm{B})$ films are similar to those discussed in previous work on $\mathrm{Ru}(\mathrm{P})$ films $[6,14]$ in order to minimize potential processing differences when comparing the films. Briefly, $\mathrm{Ru}(\mathrm{B})$ films were grown in a coldwall CVD chamber on $15 \mathrm{k} \AA \mathrm{SiO}_{2}$ deposited using tetraethyl orthosilicate on low-particle undoped Si (Sylib Wafers 812AFBA), with each substrate measuring $20 \mathrm{~mm}$ square and anchored to a stainless steel puck used to move it between chambers and position the sample as needed. The $\mathrm{Ru}$ precursor, $\mathrm{Ru}_{3}(\mathrm{CO})_{12}$ (Aldrich, 99\%), was a volatile solid-state compound distributed from a saturator into the growth chamber using $\mathrm{H}_{2}$ carrier gas, with the saturator kept

at $85{ }^{\circ} \mathrm{C}$. The Ru precursor and carrier gas was regulated to a flow rate that produced approx. 1.5 Pa partial pressure in the chamber. $\mathrm{B}_{2} \mathrm{H}_{6}$ (Voltaix, research grade) at $15 \mathrm{ppm}$ in $\mathrm{H}_{2}$ was flowed into the chamber at flow rates required to produce $\mathrm{B}_{2} \mathrm{H}_{6}$ partial pressures between 0 and $24 \mu \mathrm{Pa}$. Substrate surface temperature was maintained (at $250{ }^{\circ} \mathrm{C}$ where not otherwise specified) by a heating bulb in the stage. The CVD chamber was kept below $0.7 \mathrm{mPa}$ total pressure between growth cycles.

Plasma-assisted vapor deposition for the electrical devices used in failure testing was done in a cold-walled chamber equipped with a hand-turned rotating stage and direct current (DC) plasma ion sputtering guns (AJA International 320-2a) loaded with 99.999 at.\% pure Cu, $\mathrm{Al}$, and $\mathrm{Ru}$ targets as needed (Kurt J. Lesker \& Co) and using an $\mathrm{Ar}^{+}$plasma at $1.3 \mathrm{~Pa}$ and $100 \mathrm{~W}$ of power input (controlled with an Advanced Energy MDX 500 adjustable DC power supply). The two deposition chambers (CVD and physical vapor deposition (PVD)) and the X-ray photoelectron spectroscopy (XPS) chamber were connected by a transfer chamber that used a 
motorized cart to move the pucks on which the substrates were mounted between those three chambers, with the transfer chamber maintained at roughly $1.3 \mu \mathrm{Pa}$ when not in use.

In situ XPS was performed using a Physical Electronics 3057 XPS system with an MgKa X-ray emitter and a fixed $30^{\circ}$ angle in a third cold-walled chamber. This system included an in situ $\mathrm{Ar}^{+}$ion sputtering filament that allowed the removal of material and the construction of composition depth profiles to judge the compositional uniformity. Film thickness was calculated by measuring the attenuation of the $\mathrm{Si} 1 s$ signal in $\mathrm{Ru}$ as tabulated in the NIST database [24], with the pre-attenuation peak area regularly calibrated and sputter rates recalculated with each sample and checked for consistency with previous sputter rates. Film composition was derived by weighting the measured peak areas for the component substances by their known XPS sensitivity factors [25]: 4.273 for $\mathrm{Ru} 3 d, 0.159$ for B $1 s$, and 0.296 for C $1 s$. Because the C $1 s$ peak overlaps the $\mathrm{Ru} 3 d_{3 / 2}$ peak, those peaks were deconvoluted with freeware analytical software [26] using a Shirley background approximation with three end-points to either side of the peak, with parameters manually adjusted until the three component signal peaks reflect the known ratio of the $\mathrm{Ru} 3 d_{5 / 2}: 3 d_{3 / 2}$ peaks in pure ruthenium (1.49:1) [27]. The manual nature of the parameter re-adjustment introduces an error range of $+/-10$ at. $\%$ to the final calculated $\mathrm{C}$ content using this technique due to the low sensitivity of the $\mathrm{C} 1$ s peak compared to the $\mathrm{Ru} 3 d$ peaks.

Film thicknesses were obtained by comparing two measurements: Si $1 s$ peak attenuation calculated as noted above, and an ex situ X-ray reflectivity (XRR) measurement made using a Bruker-Nonius D8 diffractometer equipped with a sealed-tube, long fine focus $\mathrm{Cu} \mathrm{K}_{\alpha}(1.5418 \AA)$ source, a Sol-X solid state detector, and operated in the Bragg-Brentano configuration. Film roughness was measured via atomic force microscopy (AFM) (Aligent Instruments 5500). Films 
were judged to be continuous when the XPS- and XRR-derived thickness estimates matched, the XRR measurement gave a clean and definite pattern, and the AFM-based film root mean square (RMS) roughness did not exceed half of the calculated thickness. Only continuous films are included in the data reported herein.

Film crystallinity was evaluated ex situ using X-ray diffraction (XRD) (Bruker-Nonius D8) operated at glancing incidence angle $\left(0.5^{\circ}\right)$. Films were designated X-ray amorphous based on the lack of appearance peaks associated with crystalline Ru [28]. As-deposited and annealed films were studied for crystallinity. Annealing was done in the XPS chamber using a stage heating bulb under $1.3 \mathrm{mPa}$ of $\mathrm{H}_{2}$; the films were exposed to atmosphere between annealing steps due to the XRD apparatus being open to air.

The barrier testing method for copper diffusion under field stress is very similar to that outlined in Reference [7] and described in Reference [14]. $\mathrm{A} \mathrm{Cu} / \mathrm{Ru}(\mathrm{B}) / \mathrm{SiO}_{2} / \mathrm{p}-\mathrm{Si}$ stack was manufactured by growing an $\mathrm{Ru}(\mathrm{B})$ film on the $\mathrm{SiO}_{2}$ substrate, then depositing a thick $(>10 \mu \mathrm{m})$ $\mathrm{Cu}$ film on top of the film by PVD without exposing the film to atmosphere. After removing the binary film from vacuum, test devices consisting of $0.3-\mathrm{mm}$ diameter $\mathrm{Cu}$ dots on top of $1-\mathrm{mm}$ diameter $\mathrm{Ru}(\mathrm{B})$ dots were fabricated. A single $20 \mathrm{~mm} \times 20 \mathrm{~mm}$ square of wafer could produce as many as 36 device stacks for testing, but in practice about half of these were typically damaged at some point in the course of transportation and processing and failed immediately under testing, leaving 10 to 15 stacks for testing from a given film. In the failure test, a device stack was placed under a strong electric potential, and the leakage current monitored (Agilent 4156C Semiconductor Parameter Analyzer) until capacitive breakdown was observed, resulting in a sudden abrupt jump in current flow. 
Our calculations were performed using density functional theory (DFT) [29] within the Perdew-Berke-Ernzerhof generalized gradient approximation (GGA-PBE) [30] as implemented in the Vienna ab initio Simulation Package (VASP) [31]. We employed Vanderbilt-type ultrasoft pseudopotentials to describe the interaction between core and valence electrons, and a planewave basis set with a kinetic energy cutoff of $300 \mathrm{eV}$. The model structures for amorphous $\mathrm{Ru}(\mathrm{P})$ and $\mathrm{Ru}(\mathrm{B})$ were obtained using ab initio molecular dynamic (AIMD) simulations. We first created the amorphous structure of $\mathrm{Ru}$, and then replaced a given fraction of $\mathrm{Ru}$ with $\mathrm{P}$ or $\mathrm{B}$. The Ru-P or Ru-B alloy systems, each containing 72 atoms in a periodic supercell, were melted at $3500 \mathrm{~K}$ for $3 \mathrm{ps}$ with a time step of $1 \mathrm{fs}$, and then quenched to $500 \mathrm{~K}$ at a rate of $1.5 \mathrm{~K} / \mathrm{fs}$, followed by static structural optimization. Here the temperature was controlled using velocity rescaling. The structures of crystalline $\mathrm{Ru}(\mathrm{P})$ and $\mathrm{Ru}(\mathrm{B})$, as well as ruthenium, phosphorus and boron, are summarized in Table 1. For each amorphous system, 3-5 independent samples were considered to obtain good statistics in predicting its energetics and structure. The Brillouin zone integration for periodic 72-atom supercells was performed using one k-point (at Gamma) for AIMD simulations and $2 \times 2 \times 2$ Monkhorst-Pack k-point mesh [32] for geometry optimization and energy calculations. For further structural and electronic property analysis, the $\mathrm{Ru}_{80} \mathrm{P}_{20}$ and $\mathrm{Ru}_{87} \mathrm{~B}_{13}$ alloy structures were modeled using 144-atoms (consisting of $115 \mathrm{Ru}$ and $29 \mathrm{P}$ atoms in $\mathrm{Ru}_{80} \mathrm{P}_{20}, 125 \mathrm{Ru}$ and $19 \mathrm{~B}$ in $\left.\mathrm{Ru}_{87} \mathrm{~B}_{13}\right)$ in a periodic supercell volume of $2 \mathrm{~nm}^{3}$. While no simulation study has been reported for the $\mathrm{Ru}(\mathrm{P})$ and $\mathrm{Ru}(\mathrm{B})$ systems, the $\mathrm{Ni}_{80} \mathrm{P}_{20}$ structure obtained using the same procedure shows excellent agreement with that from previous AIMD simulations [33].

\section{3 - Results and Discussion:}




\section{1 - Relative stability between amorphous and crystalline $R u(P)$ and $R u(B)$ alloys}

To examine the relative stability of the amorphous versus ordered structures of $\mathrm{Ru}(\mathrm{P})$ and $R u(B)$, we calculated and compared the total energies of $R u(P)$ and $R u(B)$ supercells at varying $P$ and B contents. Here, we only considered the regions below below 30 at.\% of P and 20 at.\% of $\mathrm{B}$, where the transition from a more stable ordered to amorphous structure was found. As shown in Figure 1, the calculation results demonstrate that the amorphous $\mathrm{Ru}(\mathrm{P})$ and $\mathrm{Ru}(\mathrm{B})$ structures become energetically more favorable than their ordered counterparts when the $\mathrm{P}$ and $\mathrm{B}$ contents are above 20 at. $\%$ and 10 at.\%, respectively Here, the ordered structures were obtained by replacing $\mathrm{Ru}$ with $\mathrm{P}$ or $\mathrm{B}$, starting with the hexagonal close packed structure of pure $\mathrm{Ru}$ (with a lattice constant of $2.70 \AA$ ). Similarly, the amorphous structures were constructed by replacing Ru with $\mathrm{P}$ or $\mathrm{B}$ atoms in $a-\mathrm{Ru}$; the replacing sites were carefully chosen to ensure homogeneous distribution of $\mathrm{P} / \mathrm{B}$. Both ordered and amorphous alloys were modeled using a 72-atom supercell, and the atomic positions and the supercell volume were optimized to minimize the total energy.

Figure 2 shows the predicted volume change exhibiting a distinctive nonlinear trend for the $\mathrm{Ru}(\mathrm{P})$ alloy but a linear decrease for the $\mathrm{Ru}(\mathrm{B})$ alloy. The $\mathrm{Ru}(\mathrm{P})$ alloy volume becomes a minimum at 20 at.\%, yielding the highest packing density, while the $\mathrm{Ru}(\mathrm{B})$ alloy volume decrease, leads to increased packing density as B content increases. This is largely due to the large atomic size difference between Ru and B atoms. As expected, the crystalline phase is slightly denser than the amorphous alloy of corresponding composition. The smaller atomic size of B than P decreases the composition limit to form an amorphous structure.

For the sake of comparison, we also evaluated the crystalline-to-amorphous transition using the following universal correlation between glass formability and atomic volume ratio in a 
binary alloy [32]: $C_{\min }=0.1 /\left|\gamma^{3}-1\right|$, where $C_{\min }$ is the minimum concentration of solute element needed to produce the amorphous phase and $\gamma\left(=R_{b} / R_{a}\right)$ is the radius ratio of the solute $\left(R_{b}\right)$ to the solvent $\left(R_{a}\right)$. The elastic model has been widely adopted in predicting the structural instability of a crystalline binary alloy (as a function of solute concentration) due to the size difference between solvent and solute atoms. Taking $1.338 \AA$ [34], $1.06 \AA$ [35], and $0.82 \AA$ [36] for Ru, P, and $\mathrm{B}$ atomic radii, respectively, the required minimum concentrations for $\mathrm{P}$ (in an amorphous $\mathrm{Ru}(\mathrm{P})$ alloy) and $\mathrm{B}$ (in an amorphous $\mathrm{Ru}(\mathrm{B})$ alloy) are estimated to be 19.5 at.\% and 11.5 at.\%, respectively, which is in good agreement with our DFT calculation results. These results demonstrate that the amorphization of binary alloys is mainly driven by the elastic strain contribution arising from the atomic size difference between $\mathrm{Ru}$ and $\mathrm{P}(\mathrm{B})$ atoms.

Figure 3 shows a variation in the mixing enthalpy for amorphous and crystalline $\mathrm{Ru}(\mathrm{P})$ and $\mathrm{Ru}(\mathrm{B})$ alloys as a function of the $\mathrm{Ru}: \mathrm{P}(\mathrm{B})$ composition ratio, with respect to crystalline $\mathrm{Ru}$ $(c-\mathrm{Ru})$ and crystalline $\mathrm{P}$ or $\mathrm{B}(c-\mathrm{P}$ or $c-\mathrm{B})$. The mixing enthalpy per atom $\left(\Delta E_{m i x}\right)$ is given by:

$$
\Delta E_{m i x}=E(\text { alloy })-(1-x) E_{R u}-x E_{P(B)}
$$

where $E$ (alloy) is the total energy per atom of the $\mathrm{Ru}(\mathrm{P})$ or $\mathrm{Ru}(\mathrm{B})$ alloy examined, $x$ is the number fraction of $\mathrm{P}(\mathrm{B})$, and $E_{\mathrm{Ru}}$ and $E_{\mathrm{P}(\mathrm{B})}$ are the total energies per atom of crystalline $h c p \mathrm{Ru}$ and $\mathrm{P}(\mathrm{B})(c-\mathrm{Ru}, c-\mathrm{P}$ or $c-\mathrm{B})$, respectively. The results indicate that the $\mathrm{Ru}(\mathrm{P})$ alloy forms the most favorable structure when the P content is around 40-60 at.\%, with an energy gain of $\sim-0.3$ $\mathrm{eV} /$ atom, while the mixing enthalpy for the $\mathrm{Ru}(\mathrm{B})$ alloy gradually decreases with increasing $\mathrm{B}$ content and exhibits a minimum value of $\sim-0.2 \mathrm{eV} /$ atom around 50 at.\%. For the $\mathrm{Ru}(\mathrm{P})$ alloy, the large negative mixing enthalpies suggest that $\mathrm{P}$ atom can easily be incorporated into the amorphous Ru matrix above 20 at. \% P. This was observed experimentally in previous studies that showed amorphous $\mathrm{Ru}(\mathrm{P})$ thin films formed above approximately 17 at.\% P [14]. We also 
note that the mixing enthalpy becomes positive for low $\mathrm{P}$ content, which might indicate the presence of a barrier for incorporation of $\mathrm{P}$ into pure $\mathrm{Ru}$.

On average, for $\mathrm{Ru}(\mathrm{P})$ the total energies are $\sim 0.3 \mathrm{eV} /$ atom lower for crystalline phases than their amorphous counterparts; hence $a-\mathrm{Ru}(\mathrm{P})$ alloys may be expected to undergo crystallization at elevated temperatures. Experiments showed 3 to $5 \mathrm{~nm}$-thick films remained amorphous upon annealing at $400{ }^{\circ} \mathrm{C}$, but began to crystallize under a $450{ }^{\circ} \mathrm{C} 3$-hr anneal [14]. The $\mathrm{Ru}(\mathrm{B})$ alloy mixing enthalpy is lower for the crystalline phase than the amorphous phase between 20 and 50 at.\% B (Figure 3). The thermal stability of the amorphous $\mathrm{Ru}(\mathrm{B})$ phase is reported herein and films with 13 to 20 at.\% B remained amorphous at $450{ }^{\circ} \mathrm{C}$ and formed polycrystalline films at $500{ }^{\circ} \mathrm{C}$.

Using this thermodynamic perspective, the amorphous structure forming ability is predicted by the comparison of the mixing enthalpy and the mixing enthalpy difference between crystalline and amorphous phases. The lower the mixing enthalpy, the higher the glass forming ability, and the lower the mixing enthalpy difference between the crystalline and amorphous phases, the higher the glass forming ability. Based on this correlation, the lower mixing enthalpy and lower mixing enthalpy difference for the $\mathrm{Ru}(\mathrm{P})$ alloy than the $\mathrm{Ru}(\mathrm{B})$ alloy may enhance its glass forming ability.

To gain understanding of the structural stability between constituent atoms, we explored the atomic distribution of selected $\mathrm{Ru}_{80} \mathrm{P}_{20}$ and $\mathrm{Ru}_{87} \mathrm{~B}_{13}$ alloys as these were near the transition where amorphous alloys should form. Figure 4 shows a set of the pair distribution functions from our AIMD simulations, together with corresponding crystalline structures for comparison. The amorphous structures were characterized using the pair distribution function (PDF), $g(r)$, which is defined as [37] 


$$
g(r)=\frac{V}{N} \frac{n(r)}{4 \pi r^{2} \Delta r}
$$

where $n(r)$ represents particles in a shell within the region $r \pm \Delta r / 2$, where $\Delta r$ is the shell thickness, and $N$ denotes the number of particles in the model volume $V$.

The pair distribution function $g(r)$ was computed using 3 different 144-atom supercells for good statistics. No sharp second-neighbor peak is present, which confirms the amorphous nature (i.e., a lack of long-range order) of the $\mathrm{Ru}(\mathrm{P})$ and $\mathrm{Ru}(\mathrm{B})$ alloys. The first peak in $\mathrm{g}_{\mathrm{Ru}-\mathrm{Ru}}(\mathrm{r})$ of $a-\mathrm{Ru}_{80} \mathrm{P}_{20}$ is around $2.7 \AA$, which nearly corresponds to the bond distance of amorphous $\mathrm{Ru}$ $(2.7 \AA)$, while the first peak of $g_{\mathrm{Ru}-\mathrm{Ru}}(r)$ in $a-\mathrm{Ru}_{87} \mathrm{~B}_{13}$ is $2.6 \AA$, which indicates that the $a-\mathrm{Ru}_{87} \mathrm{~B}_{13}$ is more closely packed than $a-\mathrm{Ru}_{80} \mathrm{P}_{20}$ even at lower $\mathrm{B}$ content than $\mathrm{P}$ content.

For the pair distribution functions for $\mathrm{P}(\mathrm{B})$, the statistics for the $g_{\mathrm{P}-\mathrm{P}}(r)$ and $g_{\mathrm{B}-\mathrm{B}}(r)$ are not good comparing with the crystalline counterparts with coordination number $(\mathrm{CN})=0$, but the small intensity of a partial peak and a calculated coordination number of 0.85 (at cut-off of 3.27 $\AA$ ) and 0.44 (at cut-off of $3.03 \AA$ ) for $\mathrm{P}$ and $\mathrm{B}$, respectively, describe the existence of dimers $\left(\mathrm{P}_{2}\right.$ and $\left.\mathrm{B}_{2}\right)$. The pair distribution functions between $\mathrm{Ru}$ and $\mathrm{P}(\mathrm{B}), g_{\mathrm{Ru}-\mathrm{P}}(r)$ and $g_{\mathrm{Ru}-\mathrm{B}}(r)$, are also shown in Figure 4. The first peaks in $a-\mathrm{Ru}_{80} \mathrm{P}_{20}$ and $a-\mathrm{Ru}_{87} \mathrm{~B}_{13}$ are at $2.4 \AA$ and $2.3 \AA$, respectively. This suggests the strong intermixing between the $\mathrm{Ru}$ and $\mathrm{P}(\mathrm{B})$ atoms, namely, a strong chemical short-range order. This result is similar to the model proposed by Lee and Hwang [38] for the Au-Si alloy, which shows strong short-range order with the negative mixing enthalpy. For the total pair distribution function, the $a-\mathrm{Ru}_{80} \mathrm{P}_{20}$ and $a-\mathrm{Ru}_{87} \mathrm{~B}_{13}$ alloys show distinct amorphous character as an evidenced by the split second peak, where the average coordination number of $\mathrm{Ru}$ around $\mathrm{P}$ and $\mathrm{B}$ are 9.10 and 8.74 , respectively.

From these $g(r)$ features we can estimate that the structures of the amorphous alloys are governed by a chemical ordering effect due to the chemical interaction between the Ru and the 
$\mathrm{P}(\mathrm{B})$ atoms. More insight into the structural changes is gained by analyzing the structures and characterizing the local environment surrounding a $\mathrm{P}(\mathrm{B})$ atom by $\mathrm{Ru}$ atoms. As displayed in Table 2, we calculated the first-neighbor $\mathrm{CN}$ of the $\mathrm{Ru}$ atoms around $\mathrm{P}$ and $\mathrm{B}$ at selected $\mathrm{Ru}{ }_{80} \mathrm{P}_{20}$ and $\mathrm{Ru}_{87} \mathrm{~B}_{13}$ alloys as a function of normalized cutoff radius, $r^{*}$. With increasing $r^{*}$, the average $\mathrm{CN}$ increases. These results also show that pure $\mathrm{Ru}$ is less closely packed than the alloys. This is attributed to the relatively smaller size of $\mathrm{P}$ or $\mathrm{B}$ compared to $\mathrm{Ru}$. The $\mathrm{CN}$ of $\mathrm{Ru}_{80} \mathrm{P}_{20}$ is somewhat lower than that of $\mathrm{Ru}_{87} \mathrm{~B}_{13}$ within the nearest neighbor distance $\left(r^{*}<1.2\right)$. However, when $r^{*}$ is large (> 1.3), the $\mathrm{CN}$ of the $\mathrm{Ru}_{80} \mathrm{P}_{20}$ is somewhat greater than that of the $\mathrm{Ru}_{87} \mathrm{~B}_{13}$. The radius of $\mathrm{B}$ is smaller than that of $\mathrm{P}$; therefore the average $\mathrm{CN}$ of $\mathrm{Ru}$ around the $\mathrm{B}$ atoms within the nearest neighbor distance is lower than that of $\mathrm{Ru}$ around the $\mathrm{P}$ atoms.

\section{2 - Voronoi analysis}

The local atomic ordering of $\mathrm{Ru}(\mathrm{P})$ and $\mathrm{Ru}(\mathrm{B})$ were examined using the Voronoi tessellation method $[39,40]$. The $\mathrm{Ru}(\mathrm{P})$ or $\mathrm{Ru}(\mathrm{B})$ alloy with a moderate $\mathrm{P}$ or $\mathrm{B}$ content results in a glassy structure exhibiting a distinct topological and chemical short-range order (SRO). The type of coordination polyhedron around a $\mathrm{P}(\mathrm{B})$ atom can be specified using the Voronoi index < $i 3, i 4, i 5, i 6, \ldots>$, where $i$ indicates the number of n-edged faces of the Voronoi polyhedron and $\Sigma i n$ is the total CN, to designate and differentiate the type of coordination polyhedron surrounding the center of solute atoms. For the $a-\mathrm{Ru}_{80} \mathrm{P}_{20}$ structure (Figure 5a), the solute coordination polyhedra form the TTP (tri-capped trigonal prism packing), which corresponds to a Voronoi index of $\langle 0,3,6,0\rangle$; mono-capped square Archimedean antiprism (slightly distorted from the TTP), which corresponds to a Voronoi index of $\langle 0,5,4,0\rangle$; and CN10 polyhedra, which has a Voronoi index of $\langle 0,4,6,0\rangle$. For $a-\mathrm{Ru}_{87} \mathrm{~B}_{13}$ structure (Figure $5 \mathrm{~b}$ ), the solute coordination polyhedra form the CN8 Kasper polyhedron, with a Voronoi index of $<0,4,4,0\rangle$, and CN9 TTP, 
which corresponds to a Voronoi index of $\langle 0,3,6,0\rangle$. The dominant polyhedra of the solute in alloys are shown in Table 3. Voronoi analysis indicates that the average $\mathrm{CN}$ is 9.3 for the $\mathrm{Ru}(\mathrm{P})$ alloy, and 8.4 for the $\mathrm{Ru}(\mathrm{B})$ alloy, which corresponds to the average $\mathrm{CN}$ calculated from integration of the first peak of the pair distribution function (PDF).

It is well known that the preference polyhedra and $\mathrm{CN}$ are governed by the effective atomic size ratio, $\lambda$ between solvent and solute atoms [33]. For instance, an earlier study shows that with decreasing $\lambda$, the preferred polyhedra changes from the Frank-Kasper type (for $\lambda>1.2$ ) to the icosahedral type $(\lambda=0.902)$, and then to the bi-capped square archimedian antiprism type $(\lambda=0.835)$, and then to the TTP type $(\lambda=0.732)$. Considering the dominant $\mathrm{CN}$ of 9 , we can expect that the polyhedron with a Voronoi index of $\langle 0,3,6,0\rangle$, TTP phase found in the $\mathrm{Ru}(\mathrm{P})$ alloy is similar to that found in $\mathrm{Ni}_{81} \mathrm{~B}_{19}$, while the polyhedron, $<0,4,4,0>$ found in the $\mathrm{Ru}(\mathrm{B})$ alloy, which has the $\mathrm{CN}$ of 8 , is also a part of polyhedra found in $\mathrm{NiB}$ alloys. While no simulation study has been reported for $\mathrm{Ru}(\mathrm{P})$ and $\mathrm{Ru}(\mathrm{B})$, we also calculated the $\mathrm{Ni}_{80} \mathrm{P}_{20}$ and $\mathrm{Ni}_{80} \mathrm{~B}_{20}$ as part of the verification of $\mathrm{Ru}(\mathrm{P})$ and $\mathrm{Ru}(\mathrm{B})$. The dominant polyhedra found in the $\mathrm{Ni}_{80} \mathrm{P}_{20}$ and $\mathrm{Ni}_{80} \mathrm{~B}_{20}$ alloys are $[\langle 0,2,8,0\rangle,\langle 04,6,0\rangle,\langle 0,2,8,1\rangle]$ and $[\langle 0,3,6,0\rangle,\langle 04,4,0\rangle$, $<0,2,8,0>$ ], respectively. In addition, the average $\mathrm{CN}$ of the $\mathrm{P}$ and $\mathrm{B}$ atoms is 10.5 and 9.1 for $\mathrm{Ni}(\mathrm{P})$ and $\mathrm{Ni}(\mathrm{B})$, respectively. These are very similar to previous findings [33], that show the dominant polyhedra in $\mathrm{Ni}_{80} \mathrm{P}_{20}$ and $\mathrm{Ni}_{80} \mathrm{~B}_{20}$ are $\langle 0,2,8,0\rangle$ and $\langle 0,3,6,0\rangle$, respectively. Therefore, it is evident that our approach to elucidate the $\mathrm{SRO}$ and $\mathrm{CN}$ for $\mathrm{Ru}(\mathrm{P})$ and $\mathrm{Ru}(\mathrm{B})$ is reasonable in predicting the glasses properties as well.

As is shown in Figure 5, the formation of 'quasi-equivalent' P-centered Ru clusters arising from topological and chemical SRO is also likely to lead to the medium- range order (MRO) in the binary alloy. In fact, the short-to-medium range order is seen in other metallic 
glasses, particularly in transition metal-metalloid and transition metal-transition metal systems where the chemical short-range-order is significant [41-43]. In $\mathrm{Ni}_{80} \mathrm{P}_{20}$ [33], the $\mathrm{P}$ atom-centered clusters (Fig 5c) are packed with the icosahedral order, which is very similar to the topological configuration with the AIMD simulation. In the same manner, $\mathrm{Ru}(\mathrm{P})$ has shown the icosahedra type regardless of the type of SRO. These results indicate that the MRO found in the metalmetalloid binary alloy has the icosahedral ordering, which has the most stable packing in metallic glasses.

\section{3 - Film growth using $B_{2} H_{6}$ as the B source in $\mathrm{Ru}(\mathrm{B}) \mathrm{CVD}$}

As with the $\mathrm{PH}_{3}$ hydride used in $\mathrm{Ru}(\mathrm{P})$ film growth [14], $\mathrm{B}_{2} \mathrm{H}_{6}$ appears to decompose rapidly and completely on $\mathrm{Ru}$ and $\mathrm{Ru}(\mathrm{B})$ under the film growth conditions at $250{ }^{\circ} \mathrm{C}$. This was expected since $\mathrm{B}_{2} \mathrm{H}_{6}$ decomposes on $\mathrm{Ru}(0001)$ between 230 and $400 \mathrm{~K}$ depending on the $\mathrm{B}_{2} \mathrm{H}_{6}$ exposure level [23]. Unexpectedly, much higher B incorporation levels were found in the films grown with $\mathrm{B}_{2} \mathrm{H}_{6}$ when compared to the $\mathrm{P}$ incorporation levels found with the same $\mathrm{PH}_{3}$ partial pressure. In practical terms the high reactivity of $\mathrm{B}_{2} \mathrm{H}_{6}$ on $\mathrm{Ru}$ presents challenges to limit the $\mathrm{B}$ incorporation levels during film during growth. The $15 \mathrm{ppm} \mathrm{B}_{2} \mathrm{H}_{6}$ in $\mathrm{H}_{2}$ concentration used herein was the lowest concentration commonly sold by the manufacturer. Control of film composition also relied on $\mathrm{B}_{2} \mathrm{H}_{6}$ source gas flow rate adjustments during growth. Other $\mathrm{CVD}$ chambers will need to establish protocols for limiting and regulating the $\mathrm{B}_{2} \mathrm{H}_{6}$ partial pressure to very low levels.

In another similarity to $\mathrm{Ru}(\mathrm{P})$ films grown with $\mathrm{PH}_{3}[14], \mathrm{B}$ accumulates on the growth surface more rapidly than it can be incorporated by the growing $\mathrm{Ru}(\mathrm{B})$ film, resulting in a steeply increasing $\mathrm{B}$ concentration within the film from the $\mathrm{Ru}(\mathrm{B}) / \mathrm{SiO}_{2}$ starting interface to the growth 
surface if the $\mathrm{B}_{2} \mathrm{H}_{6}$ partial pressure is kept static during growth. To keep the film composition uniform with thickness the $\mathrm{B}_{2} \mathrm{H}_{6}$ partial pressure in the CVD chamber was varied over the course of the growth cycle for each film, beginning high and then gradually being stepped down to zero over the course of film growth (shown in Figure 6 for a film grown at $250{ }^{\circ} \mathrm{C}$ ). Figure 7 presents the concentration profile corresponding to the pressure settings in Figure 6 with the position based on sputtering times as described in Section 2. There is less than 5 at.\% variation in the calculated B content of the film over three nm of total film thickness. The specifics of the concentration step sizes and times were based on previous $\mathrm{Ru}(\mathrm{P}) \mathrm{CVD}$ studies with $\mathrm{PH}_{3}[14]$ and were found to produce a low variation in the mean B concentration with position relative to the $\mathrm{Ru}(\mathrm{B}) / \mathrm{SiO}_{2}$ interface. Overall film composition is reported using a mean $\mathrm{B}$ concentration.

Films were grown at substrate temperatures of $250{ }^{\circ} \mathrm{C}, 300{ }^{\circ} \mathrm{C}$, and $350{ }^{\circ} \mathrm{C}$, and no dependence in B the concentration profile was observed within this temperature range. This was expected since $\mathrm{B}_{2} \mathrm{H}_{6}$ decomposes on $\mathrm{Ru}$ at a much lower temperature than the lower limits of the $\mathrm{Ru}_{3}(\mathrm{CO})_{12}$ ruthenium precursor deposition temperature $\left(150^{\circ} \mathrm{C}\right)[44]$. A substrate temperature of $250^{\circ} \mathrm{C}$ was used for all films reported herein.

The carbon content of the films was monitored with XPS, and as with the $\mathrm{Ru}(\mathrm{P})$ films from $\mathrm{PH}_{3}$ [14] the $\mathrm{Ru}(\mathrm{B}) \mathrm{CVD}$ films were found to have $\mathrm{C}$ contents between 0 and 10 at.\%. The likely source of $\mathrm{C}$ is the carbonyl ligands in the $\mathrm{Ru}_{3}(\mathrm{CO})_{12}$ precursor.

An important concern for $\mathrm{Ru}(\mathrm{B})$ alloy films in a $\mathrm{Cu}$ diffusion barrier application, is the stability of the amorphous phase. The first-principles predictions (Figure 1) suggest thin $\mathrm{Ru}(\mathrm{B})$ films will be amorphous above 10 at.\% B and polycrystalline below 10 at.\%. The low sensitivity of the B $1 s$ signal in XPS makes it difficult to quantify concentrations below 10 at.\% B whereas concentrations above 10 at. $\%$ B can be quantified with an estimated uncertainty of $+/-2.5$ at. $\%$ 
B. Almost all $\mathrm{Ru}(\mathrm{B})$ films in the $10-15$ at.\% $\mathrm{B}$ range were $\mathrm{X}$-ray amorphous as grown, and films above 15 at.\% B were amorphous (Figure 8). Ru(B) films below the 10 at.\% B threshold were found to be polycrystalline, exhibiting the XRD features of pure Ru.

Figure 8 presents the resistivity of $3-\mathrm{nm} \mathrm{Ru}(\mathrm{B})$ films obtained by measuring sheet resistance of films with a 4-point probe). There is no discernible trend in resistivity with B content, with the highest resistivity recorded at $120 \mu \Omega-\mathrm{cm}$. These values are similar to those of $\mathrm{Ru}(\mathrm{P})$ films below 25 at.\%P in reference [14]. These results are close to the resistivity of singlecrystalline Ru films of comparable thickness (50 to $80 \mu \Omega$-cm), which are higher than bulk Ru due to the effects of dimensional restriction $[45,46]$.

XPS analysis of the $\mathrm{Ru}(\mathrm{B})$ films was done in situ, but often following several hours of cooling and subsequent transfer to the analysis chamber under vacuum following growth. XPS $\mathrm{Ru} 3 d$ peaks were consistent with zero-valent ruthenium. In addition to the zero-valent B 1 s peak at $189.4 \mathrm{eV}$, a secondary boron peak was often observed at $193 \mathrm{eV}$, which is consistent boron oxides, This 193-eV signal disappears following any amount of sputtering (as little as $0.05 \mathrm{~nm}$ equivalent of sputter time) so the oxidation is limited to the boron on the film surface.

Atomic force microscopy was used to measure the roughness of various $3 \mathrm{~nm}$-thick amorphous $\mathrm{Ru}(\mathrm{B})$ films (not shown). RMS roughness of continuous films was approximately $0.1 \mathrm{~nm},+/-0.05 \mathrm{~nm}$ on average, which is similar to amorphous $\mathrm{Ru}(\mathrm{P})$ films of comparable thickness [14]. The AFM technique was primarily used to check film continuity. Attempts to produce films less than $3 \mathrm{~nm}$ in thickness resulted in discontinuous films with large RMS roughnesses indicating that the films were not continuous.

Temperature stability of the $\mathrm{Ru}(\mathrm{B})$ amorphous films was evaluated by alternating 3-hr anneals of the $\mathrm{Ru}(\mathrm{B})$ films with $\mathrm{X}$-ray diffraction analysis. Four films were tested, all 3-nm 
$\mathrm{Ru}(\mathrm{B})$ films were grown at $250{ }^{\circ} \mathrm{C}$, one with 13 at.\% $\mathrm{B}$, two with 15 at.\% $\mathrm{B}$, and one with 20

at.\% B. Each film was amorphous as-grown, and checked for crystallization using XRD

following a $300{ }^{\circ} \mathrm{C}$ anneal, then a 400,450 , and $500{ }^{\circ} \mathrm{C}$ anneal in sequence. All films remained amorphous through the first three anneal cycles, and all films exhibited diffraction features associated with the Ru crystal structure following the $500{ }^{\circ} \mathrm{C}$ anneal as illustrated in Figure 9 for the 20 at.\% B film. This is similar to the crystallization of $3 \mathrm{~nm} \mathrm{Ru(P)}$ amorphous films, which were found to occur at $450{ }^{\circ} \mathrm{C}$ following a similar anneal sequence [14].

\section{4 - Barrier performance of $R u(B)$ and comparison to amorphous $R u(P)$}

The electrical failure test described in Experimental Methods was performed on several 3 $\mathrm{nm} \mathrm{Ru}(\mathrm{B})$ films, to provide a direct comparison to the performance of previously-studied $\mathrm{Ru}(\mathrm{P})$ ultrathin films and a 5-nm Ta/TaN stack produced using in-house PVD in the study in reference [7]. Further discussion of the method and previous related work can be found in several of the references [7, 14, 47]. Three films were tested: a 15-at.\% B Ru(B) film a similar 15-at. \% B $\mathrm{Ru}$ (B) film plated with an additional $2 \mathrm{~nm}$ of PVD Ru to bring its overall thickness up to the 5 $\mathrm{nm}$ to allow comparison with $\mathrm{Ru}(\mathrm{P})$ results $[7,14]$, and a 3-nm 25 at.\% $\mathrm{B} \mathrm{Ru}(\mathrm{B})$ film to provide an idea of whether increasing $\mathrm{B}$ content beyond the point where $\mathrm{Ru}(\mathrm{B})$ grows as an amorphous film provides any benefit to the possible $\mathrm{Cu}$ diffusion barrier application.

Figure 10 shows the measured times to failure for the 3-nm 15 -at.\% B and 25 -at.\% B $\mathrm{Ru}(\mathrm{B})$ films. Figure 11 contrasts the average failure times for the 15 -at.\% $\mathrm{B} \mathrm{Ru(B)}$ film to those of a 3-nm amorphous $\mathrm{Ru}(\mathrm{P})$ film and a 5-nm CVD Ru film grown without alloying elements [14], and a 5-nm TaN film grown in situ for benchmarking in previous work [7]. Both amorphous Ru alloy films perform significantly better than pure CVD Ru despite lower thickness, indicating that amorphous phases mitigate the issue of grain boundary diffusion as 
predicted and demonstrating that both $\mathrm{B}$ and $\mathrm{P}$ alloys with $\mathrm{Ru}$ can serve in the role of $\mathrm{Cu}$ diffusion barrier/liner layer in interconnect devices.

\section{4 - Summary and conclusions:}

The ab initio molecular dynamics (AIMD) study shows that $\mathrm{Ru}(\mathrm{P})$ and $\mathrm{Ru}(\mathrm{B})$ alloys with moderate $\mathrm{P}(\mathrm{B})$ content can result in glassy structure exhibiting the topological and strong chemical short-to-medium range order. Amorphous phases above $\sim 20$ at. $\%$ of $\mathrm{P}$ and $\sim 10$ at. $\%$ of $\mathrm{B}$ are found to be energetically more favorable than the crystalline counterparts for $\mathrm{Ru}(\mathrm{P})$ and $\mathrm{Ru}$ (B) alloys, respectively. In the $\mathrm{Ru}_{80} \mathrm{P}_{20}$ structure, the P-centered polyhedra prefer the TTP phase with Voronoi index $\langle 0,3,6,0\rangle$, while in the $\mathrm{Ru}_{87} \mathrm{~B}_{13}$, the B-centered polyhedra prefer the $<0,4,4,0>$. In addition, the Ru-P and Ru-B systems show the icosahedral medium range ordering arising from packing the 'qusai-equivalent' P-centered (B-centered) clusters in three dimensional spaces.

Growth studies employed $\mathrm{B}_{2} \mathrm{H}_{6}$ to grow $a-\mathrm{Ru}(\mathrm{B})$ films with $\mathrm{CVD}$ and showed that it should perform well as an ultrathin $\mathrm{Cu}$ diffusion barrier. The films were amorphous above 10 at. $\% \mathrm{~B}$, consistent with the first principles predictions. Film resistivity is $<120 \mu \Omega$-cm and approaches that of single-crystalline pure $\mathrm{Ru}$ at similar thicknesses, and $\mathrm{C}$ contamination is consistently low. Consistent continuity and smoothness at $3 \mathrm{~nm}$ thickness suggest that the material is amenable to conformal deposition. Performance under failure testing shows that $a$ $\mathrm{Ru}(\mathrm{B})$ serves as a $\mathrm{Cu}$ diffusion barrier, while stability under annealing studies to $450{ }^{\circ} \mathrm{C}$ indicates that $a-\mathrm{Ru}(\mathrm{B})$ is stable under most processing conditions. Time-to-failure performance under field stress equals or exceeds previously studied $a-\mathrm{Ru}(\mathrm{P})$ amorphous films and exceed the measured time to failure of $5 \mathrm{~nm}$ films of pure Ru by $4-5$ orders of magnitude. Overall, $a-\mathrm{Ru}(\mathrm{B})$ 
amorphous films produced using the hydride precursor appear promising as a liner material in technology node sizes requiring liners at $<5 \mathrm{~nm}$ thickness.

\section{5 - Acknowledgements:}

This work was supported by the National Science Foundation (CBET-0854345 and CBET1160195). 
References:

[1] A. E. Kaloyeros, E. Eisenbraun, Ultrathin diffusion barriers/liners for gigascale copper metallization, Annu. Rev. Mater. Sci. 30 (2000) 363-385.

[2] B-S. Nguyen, J-F. Lin, D-C. Perng, 1-nm-thick graphene tri-layer as the ultimate copper diffusion barrier, App. Phys. Letters 104 (2014) 082105.

[3] K. Holloway, P. Fryer, C. Cabral, J. M. E Harper, P. J. Bailey, Tantalum as a diffusion barrier between copper and silicon: Failure mechanism and effect of nitrogen additions, J. Appl. Phys 71 (1992) $5433-5444$.

[4] L. Y. Yang, D. H. Zhang, C. Y. Li, P. D. Foo, Comparative study of Ta, TaN and Ta/TaN bilayer barriers for Cu-ultra low-k porous polymer integration, Thin Solid Films 462-463 (2004) $176-181$.

[5] N. Tsyntsaru, G. Kaziukaitis, C. Yang, H. Cesiulis, H. G. G. Philipsen, M. Lelis, J.-P. Celis, Co-W nanocrystalline electrodeposits as barrier for interconnects, J Solid State Electrochem 18 (2014) 3057-3064.

[6] J. Shin, A. Waheed, W. A. Winkenwerder, H-W. Kim, K. Agapiou, R. A. Jones, G. S. Hwang, J. G. Ekerdt, Chemical Vapor Deposition of Amorphous Ruthenium-Phosphorus Alloy Films, Thin Solid Films 515 (2007) 5298-5307.

[7] L. B. Henderson, J. G. Ekerdt, Time-to-failure Analysis of $5 \mathrm{~nm}$ Amorphous $\mathrm{Ru}(\mathrm{P})$ as a Copper Diffusion Barrrier, Thin Solid Films 517 (2009) 1645-1649.

[8] H. Kim, C. Detavenier, O van der Straten, S. M. Rossnagel, A. J. Kellock, D. G. Park, Robust $\mathrm{TaN}_{\mathrm{x}}$ diffusion barrier for $\mathrm{Cu}$-interconnect technology with subnanometer thickness by metalorganic plasma-enhanced atomic layer deposition, J. App. Phys. 98 (2005), 014308. 
[9] M. Damayanti, T. Srithatan, S. G. Mhaisalkar, Z. H. Gan, Effects of dissolved nitrogen in improving barrier properties of ruthenium, App. Phys. Letters 88 (2006) 044101.

[10] S-H Kim, K. T. Nam, A. Datta, K-B. Kim, Failure mechanism of a multilayer (TiN/Al/TiN) diffusion barrier between copper and silicon, J. App Phys 92 (2002) 5512-5519.

[11] C.-C. Yang, S. Cohen, T. Shaw, P.-C. Wang, T. Nogami, D. Edelstein, Characterization of "Ultrathin-Cu"/Ru(Ta)/TaN Liner Stack for Copper Interconnects, IEEE Electron. Dev. Lett. 31 (2010) 722-724.

[12] L. Djomeni, T. Mourier, S. Minoret, S. Fadloun, F. Piallat, S. Burgess, A. Price, Y. Zhou, C. Jones, D. Mathiot, S. Maitrejean, Study of low temperature MOCVD deposition of TiN barrier layer for copper diffusion in high aspect ratio through silicon vias, Microelectronic Eng. 120 (2014) 127-132.

[13] J. Yu, J. Bian, L. Jiang, Y. Qiu, W. Duan, F. Meng, Z. Liu Co-W nanocrystalline electrodeposits as barrier for interconnects, ECS Solid State Lett. 3(6) (2014) N15-N17. [14] D. Bost, J. G. Ekerdt, Chemical vapor deposition of ruthenium-phosphorus alloy thin films: Using Phosphine as the phosphorus source, Thin Solid Films 558 (2014) 160-164.

[15] J-H. Park, D-S. Han, Y-J. Kang, S-R. Shin, H-T. Jeon, J-W. Park, Self-forming VOx layer as $\mathrm{Cu}$ diffusion barrier for low-k dielectrics, Surface and Coat. Tech. 259 (2014) 252-256.

[16] T. Lin, H. Cheng, T. Chin, C. Chiu, J. Fang, 5-nm-thick TaSiC amorphous films stable up to $750{ }^{\circ} \mathrm{C}$ as a diffusion barrier for copper metallization, Appl. Phys. Lett. 91 (2007) 152908.

[17] S. Rawal, D. P. Norton, T. J. Anderson, L. McElwee-White, Properties of W-Ge-N as a diffusion barrier material for Cu, Appl. Phys. Lett. 87 (2005) 111902.

[18] K. Barmak, C. Cabral, K. P. Rodbell, J. M. E. Harper, On the use of alloying elements for Cu interconnect applications, J. Vac. Sci. Technol. B 24(6) (2006) 2485-2498. 
[19] I. Goswami, R. Laxman, Transition metals show promise as copper barriers, Semiconductor Internatl. 27 (2004) 49-54.

[20] H. Kim, C. Cabral, C. Lavoie, S. M. Rossnagel, Diffusion barrier properties of transition metal thin films grown by plasma-enhanced atomic-layer deposition, J. Vac. Sci. Technol. B 20 (2002) 1321-1326.

[21] K-Y. Mun, T-E. Hong, T. Cheon, Y. Jang, B-Y. Lim, S. Kim, S-H. Kim, The effects of nitrogen incorporation on the properties of atomic layer deposited Ru thin films as a directplateable diffusion barrier for $\mathrm{Cu}$ interconnect, Thin Solid Films 562 (2014) 118-125. [22] J. Shin, H-W. Kim, G. S. Hwang, J. G. Ekerdt, Time-to-failure Analysis of 5 nm Amorphous Ru(P) as a Copper Diffusion Barrrier, Surf. Coat. Technol. 201 (2007) 9256-9259. [23] J. Rodriguez, C. Truong, J. Corneille, D. W. Goodman, Molecular precursors to boron nitride thin films. 1. Adsorption of diborane on ruthenium(0001), ammonia/ruthenium(0001), and oxygen/ruthenium(0001) surfaces, J. Phys Chem, 96 (1992) 334-341.

[24] NIST X-ray Photoelectron Spectroscopy Database, 4.1 (NIST, Gaithersburg, 2012).

[25] C. D Wagner, L. E. Davis, M. V. Zeller, J. A. Taylor, R. H. Raymond, L. H. Gale, Empirical atomic sensitivity factors for quantitative analysis by electron spectroscopy for chemical analysis, Surf. Interface Anal. 3 (1981) 211-25.

[26] R. Kwok, XPSPEAK 4.1, UK Surface Analysis Forum, 2010, http://www.uksaf.org/software.html.

[27] A. V. Naumkin, A. Kraut-Vass, S. W. Gaarenstroom, C. J. Powell, NIST Standard Reference Database 20, Version 4.1, http://srdata.nist.gov/xps/Default.aspx. [28] W. Obrowski, B-Ru Binary Alloy Phase Diagrams, II Ed., in: T. B. Massalski (Ed.) ASM International, Materials Park, Ohio 1990, pp. 527 - 529. 
[29] P. Hohenberg, W. Kohn, Inhomogenous Electron Gas, Phys. Rev. 136 (1964) B864.

[30] J. P. Perdew, K. Burke, M. Ernzerhof, Generalized Gradient Approximation Made Simple, Phys. Rev. Lett. 77 (1996) 3865.

[31] G. Kresse, J. Hafner, Ab initio molecular dynamics for liquid metals, Phys. Rev. B 47 (1993) RC558.

[32] H. J. Monkhorst, J. D. P Special points for Brillouin-zone integrations, Phys. Rev. B 13 (1976) 5188.

[33] H. W. Sheng, W. K. Luo, F. M. Alamgir, J. M. Bai, E. Ma, Atomic packing and short-tomedium range order in metallic glasses, Nature 439 (2006) 419.

[34] Kynoch Press, Internl. Tables for X-ray Crystallography, Birmingham, England, 1968.

[35] J. L. C. Daams, P. Villars, J. H. N. van Vucht, Atlas of Crystal Structure Types for Intermetallic Phases, vol. 1-4, ASM International, Materials Park, OH, 1991.

[36] M. Winter, WebElements ${ }^{\mathrm{TM}}$ Periodic Table, Professional Edition, http://www. webelements.com, University of Sheffield. UK (2000).

[37] M. P. Allen, D. J. Tildesley, Computer simulation of liquids, Oxford University Press, New York, 1987, pp.54.

[38] S-H. Lee, G. S. Hwang, Structure, energetics, and bonding of amorphous Au-Si alloys, J. Chem. Phys. 127 (2007) 224710.

[39] J. L. Pinney, Random Packings and the Structure of Simple Liquids. I. The Geometry of Random Close Packing, Proc. R. Soc. A 319 (1970) 479.

[40] J. L. Pinney, Modelling the structures of amorphous metals and alloys, Nature 266 (1977) 309. 
[41] P. H. Gaskell, Amorphous Metals, Models for Structure of Amorphous Metals, in: H.

Matyja, P. G. Zielinski (Eds.), World Scientific Publishing, Singapore, 1985, pp. 35-57.

[42] P. Lamparter, Reverse monte carlo simulations of amorphous $\mathrm{Ni}_{80} \mathrm{P}_{20}$ and $\mathrm{Ni}_{81} \mathrm{P}_{19}$, Phys. Scr. T57 (1995) 72-78.

[43] W. K. Luo, H. W. Sheng, F. M. Alamgir, J. M. Bai, J. H. He, E. Ma, Icosahedral ShortRange Order in Amorphous Alloys, Phys. Rev. Lett. 92 (2004) 145502.

[44] H. Kim, S-H. Lee, W.T. Lee, S. Ganguli, H-C. Ha, S. H. Yu, Chemical vapor deposition (cvd) of ruthenium films and applications for same, US Patent 20130146468 (2013) 2

[45] H. Li, D. B. Farmer, R. G. Gordon, Y. Lin, J. Vlassak, Vapor Deposition of Ruthenium from an Amidinate Precursor, J. Electrochem. Soc. 154(12) (2007) D642-47.

[46] K. Fuchs, N. F. Mott, The conductivity of thin metallic films according to the electron theory of metals, Proc. Cambridge Philos. Soc., 34(01) (1938), 100-108.

[47] G. Raghavan, C. Chiang, P. B. Anders, S-M Tzeng, R. Villasol, G. Bai, M. Bohr, D. B. Fraser, Diffusion of copper through dielectric films under bias temperature stress. Thin Solid Films, Thin Solid Films 262 (1995) 168-76. 


\section{List of Tables}

Table 1: Equilibrium lattice parameters for crystalline $\mathrm{Ru}(\mathrm{P})$ and $\mathrm{Ru}(\mathrm{B})$ alloys obtained from GGA calculations.

Table 2: Average coordination number of $\mathrm{P}$ and $\mathrm{B}$ atoms as a function of cutoff radius. Here, the cutoff radius $\left(r^{*}\right)$ is normalized with respect to $2.4 \AA$, which was the average nearest neighbor $\mathrm{Ru}-\mathrm{P}$ distance in the $\mathrm{Ru}(\mathrm{P})$ alloy considered.

Table 3: The dominant polyhedra of the solute atoms in the metallic alloy. The values are the fraction of solute atoms in the metallic alloys and only the polyhedra relevant to our work are shown. 


\section{List of Figures}

Figure 1. Variations in total energy per atom for amorphous and ordered $\mathrm{Ru}(\mathrm{P})$ and $\mathrm{Ru}(\mathrm{B})$ alloys as a function of $\mathrm{P}(\mathrm{B})$ content (at.\%). For each amorphous structure, the reported average value was obtained from three independent 74-atom supercell calculations.

Figure 2. Variation in volume for $a-\mathrm{Ru}(\mathrm{P})$ and $a-\mathrm{Ru}(\mathrm{B})$ alloys as a function of $\mathrm{P}(\mathrm{B})$ content (at.\%). For each amorphous alloy, the reported average value was obtained from three independent 64-atom supercell calculations.

Figure 3. Variation in mixing enthalpy for amorphous and crystalline $\mathrm{Ru}(\mathrm{P})$ and $\mathrm{Ru}(\mathrm{B})$ alloys as a function of $\mathrm{P}(\mathrm{B})$ content $(x)$ in $\mathrm{Ru}_{1-x} \mathrm{P}(\mathrm{B})_{x}$.

Figure 4. Pair distribution functions $g(r)$ for (a) $a-\mathrm{Ru}_{80} \mathrm{P}_{20}$ and (b) $a-\mathrm{Ru}_{87} \mathrm{~B}_{13}$ alloys. For each plot, the average $\mathrm{CN}$ with corresponding cut-off radius in parenthesis is shown.

Figure 5. The packing of the solute-centered quasi-equivalent clusters for (a) $\mathrm{Ru}_{80} \mathrm{P}_{20}$, (b) $\mathrm{Ru}_{87} \mathrm{~B}_{13}$, and (c) $\mathrm{Ni}_{80} \mathrm{~B}_{20}$ alloys. $\mathrm{Ru}, \mathrm{Ni}, \mathrm{P}$, and $\mathrm{B}$ atoms are depicted by dark green, light green, purple, and red balls, respectively.

Figure 6. Partial pressure of diborane versus growth time for a film grown at $250{ }^{\circ} \mathrm{C}$.

Figure 7. Boron concentration profile within a film grown at $250^{\circ} \mathrm{C}$. This is the composition profile obtained using the diborane pressure changes presented in Figure 6.

Figure 8: Film resistivity versus B concentration. Solid symbols denote X-ray amorphous films.

Figure 9 X-ray diffraction spectra for a 20-at\% B film that was annealed for $3 \mathrm{hr}$ sequentially at $300,400,450$ and then $500{ }^{\circ} \mathrm{C}$. 
Figure 10:-Median time-to-failure for $3 \mathrm{~nm} \mathrm{Ru(B)}$ films, tested to dielectric failure under the listed field stress.

Figure 11: Comparison of the median time-to-failure for the 3-nm thick 15 at.\% $\mathrm{Ru}(\mathrm{B})$ film ( $\square$ ), a 3-nm thick 19 at.\% Ru(P) film as reported in Ref. [14] (०), a 5-nm-thick crystalline Ru film (•) [14], and a 5 nm TaN film (ロ) presented in Ref. [7]. 
Table 1: Equilibrium lattice parameters for crystalline $\mathrm{Ru}(\mathrm{P})$ and $\mathrm{Ru}(\mathrm{B})$ alloys obtained from GGA calculations.

\section{$\underline{\text { Lattice constants }(\AA)}$}

$\mathrm{Ru}$

P6-3/mmc(194)

P

B

$\mathrm{Ru}_{2} \mathrm{P} \quad \operatorname{Pnma}(62)$

RuP Pnma(62)

$\mathrm{RuP}_{2} \quad \mathrm{Pmmn}(59)$

$\mathrm{Ru}_{23} \mathrm{~B}_{6} \quad \mathrm{Fm}-3 \mathrm{~m}(225)$

$\mathrm{Ru}_{3} \mathrm{~B} \quad \mathrm{I}-4(82)$

$\mathrm{Ru}_{7} \mathrm{~B}_{3} \quad \mathrm{Cmc2-1(36)}$

$\mathrm{Ru}_{11} \mathrm{~B}_{8} \quad \operatorname{Pbam}(55)$

RuB Amm2(38)

$\mathrm{Ru}_{2} \mathrm{~B}_{3} \quad \mathrm{Cmcm}(63)$

$\mathrm{RuB}_{2} \quad \mathrm{Pmmn}(59)$ $a=2.732, c=4.290, \gamma=120$

$a=9.274, b=9.260, c=24.159, \beta=105.74$

$a=10.738, b=11.385, c=26.249$

$a=5.595(5.902), b=3.968(3.859)$, $c=7.181(6.896)$

$a=5.553(5.52), b=3.968(3.859)$, $c=7.181(6.896)$

$a=5.140(5.117), b=4.526(5.893)$, $c=3.923(2.871)$

$$
a=11.319
$$

$a=9.17, c=4.59$

$$
a=7.519, c=4.749, \gamma=120
$$

$a=11.673, b=11.940, c=2.886$

$a=2.877, c=2.867, \gamma=120$

$a=2.924, c=12.833, \gamma=120$ $a=4.675, b=2.879, c=4.051$ $\underline{\text { Volume }\left(\AA^{3}\right)} \quad \underline{\text { K-pts }} \quad$ \#Ru $\quad \underline{\# P(B)}$

27.65

2009.85

1062.56

159.43

$\begin{array}{lll}64 & 8 & 4\end{array}$

110.01

$\begin{array}{lll}216 & 4 & 4\end{array}$

91.26

$\begin{array}{lll}216 & 2 & 4\end{array}$

362.68

$216 \quad 23 \quad 6$

195.19

$\begin{array}{lll}105 & 12 & 4\end{array}$

233.65

$\begin{array}{lll}216 & 14 & 6\end{array}$

399.45

27

$22 \quad 16$

81.10

216

95.70

21646

81.10

$\begin{array}{lll}216 & 2 & 4\end{array}$ 
Table 2: Average coordination number of $\mathrm{P}$ and $\mathrm{B}$ atoms as a function of cutoff radius. Here, the cutoff radius $\left(r^{*}\right)$ is normalized with respect to $2.4 \AA$, which was the average nearest neighbor $\mathrm{Ru}-\mathrm{P}$ distance in the $\mathrm{Ru}(\mathrm{P})$ alloy considered.

$\underline{\text { Alloy }}$

basis atom

Average Coordination number

$\begin{array}{lcccccc} & & \underline{\mathbf{r}^{*}=\mathbf{1 . 1}} & \underline{\mathbf{1 . 2}} & \underline{\mathbf{1 . 3}} & \underline{\mathbf{1 . 4}} & \underline{\mathbf{1 . 5}} \\ \alpha-\mathrm{Ru} & \mathbf{R u} & 5.0 & 8.6 & 10.9 & 12.2 & 12.5 \\ \alpha-\mathrm{Ru}_{80} \mathrm{P}_{20} & \mathbf{P} & 3.6 & 9.1 & 11.8 & 12.7 & 13.6 \\ \alpha-\mathrm{Ru}_{87} \mathrm{~B}_{13} & \mathbf{B} & 4.3 & 9.4 & 10.9 & 12.1 & 13.2\end{array}$


Table 3: The dominant polyhedra of the solute atoms in the metallic alloy. The values are the fraction of solute atoms in the metallic alloys and only the polyhedra relevant to our work are shown.

\begin{tabular}{lcccccc} 
Voronoi index & $\langle\mathbf{0 , 4 , 4 , 0}\rangle$ & $\mathbf{< 0 , 3 , 6 , 0}$ & $\langle\mathbf{0 , 5 , 4 , 0}$ & $\langle\mathbf{0 , 2 , 8 , 0}$ & $\langle\mathbf{0 , 4 , 6 , 0}\rangle$ & $\langle\mathbf{0 , 2 , 8 , 1}\rangle$ \\
\hline$a-\mathrm{Ru}_{80} \mathrm{P}_{20}$ & 0.05 & 0.24 & 0.24 & - & 0.24 & - \\
$a-\mathrm{Ru}_{87} \mathrm{~B}_{13}$ & 0.6 & 0.4 & - & - & - & - \\
$a-\mathrm{Ni}_{80} \mathrm{P}_{20}$ & - & - & - & 0.7 & 0.1 & 0.1 \\
$a-\mathrm{Ni}_{80} \mathrm{~B}_{20}$ & 0.21 & 0.43 & 0.07 & 0.21 & - & -
\end{tabular}


Figure 1
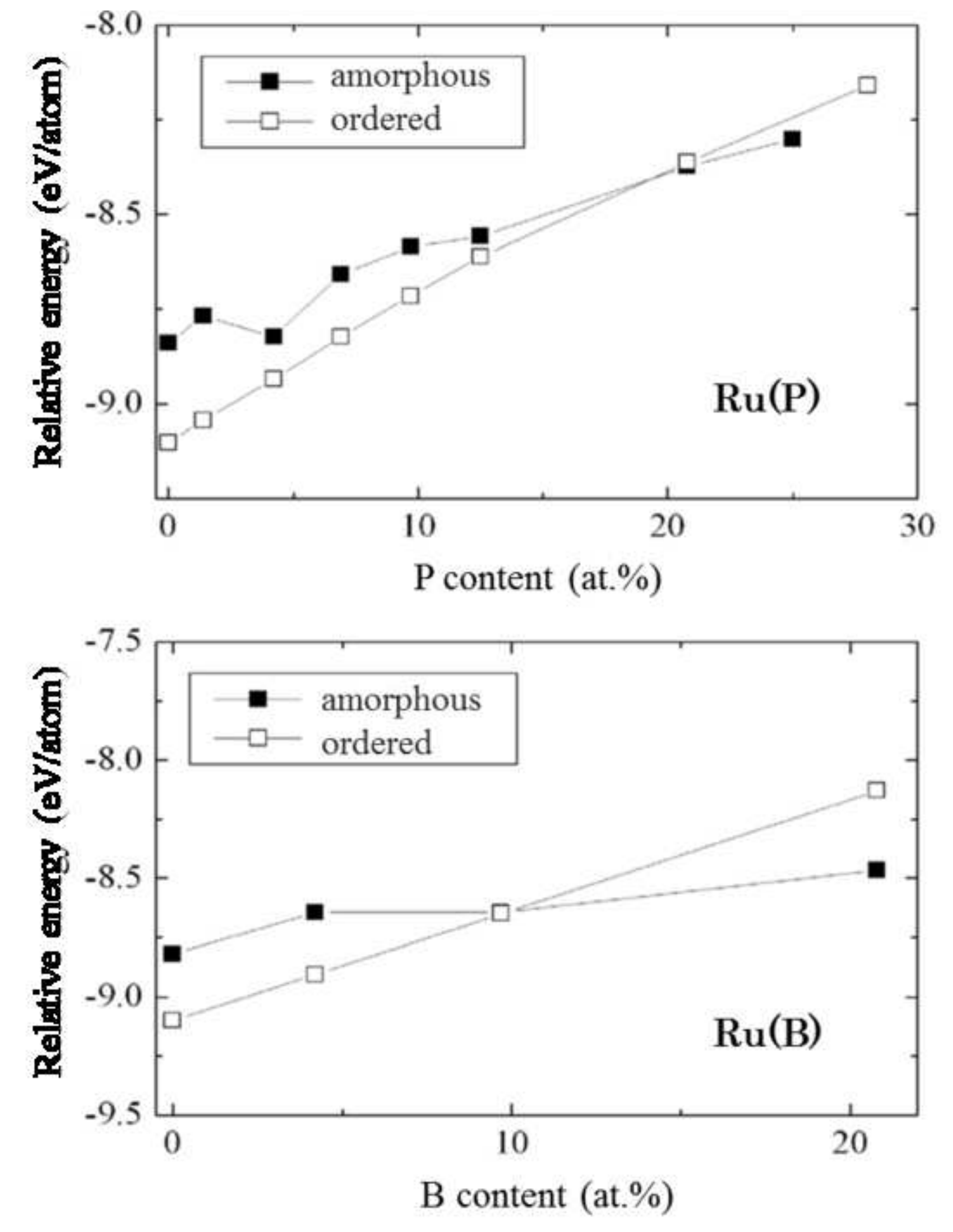

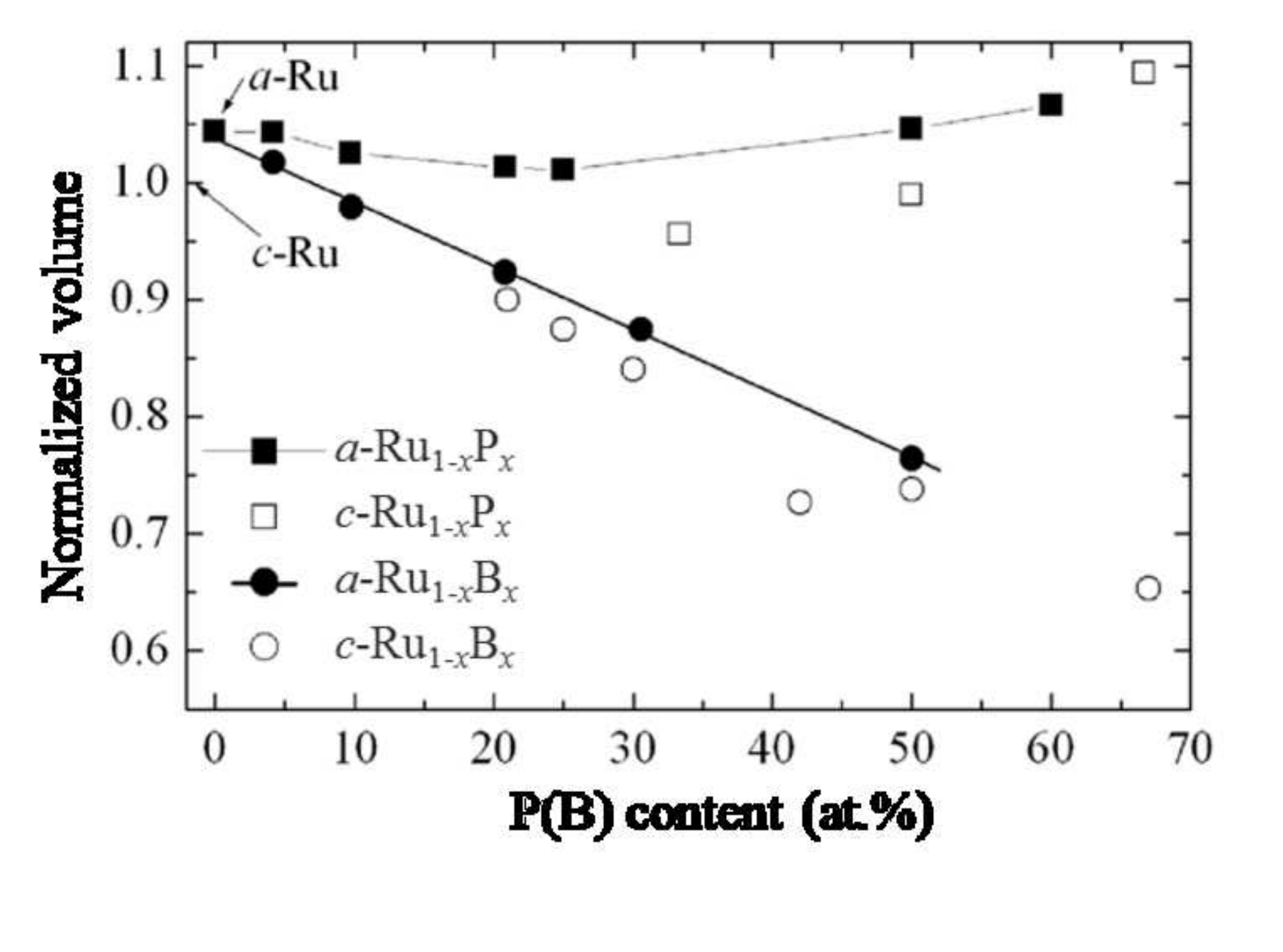


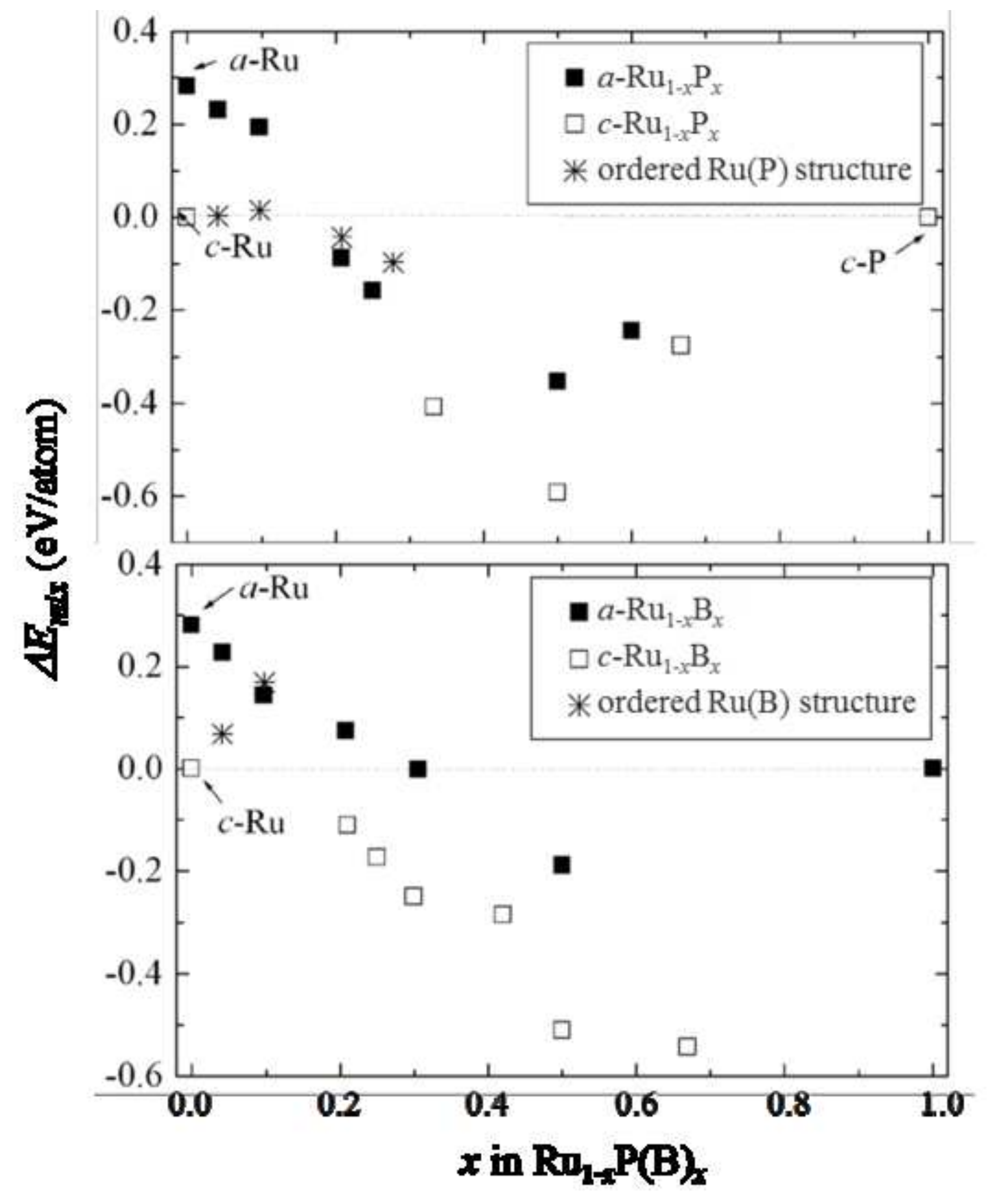

Figure 3 

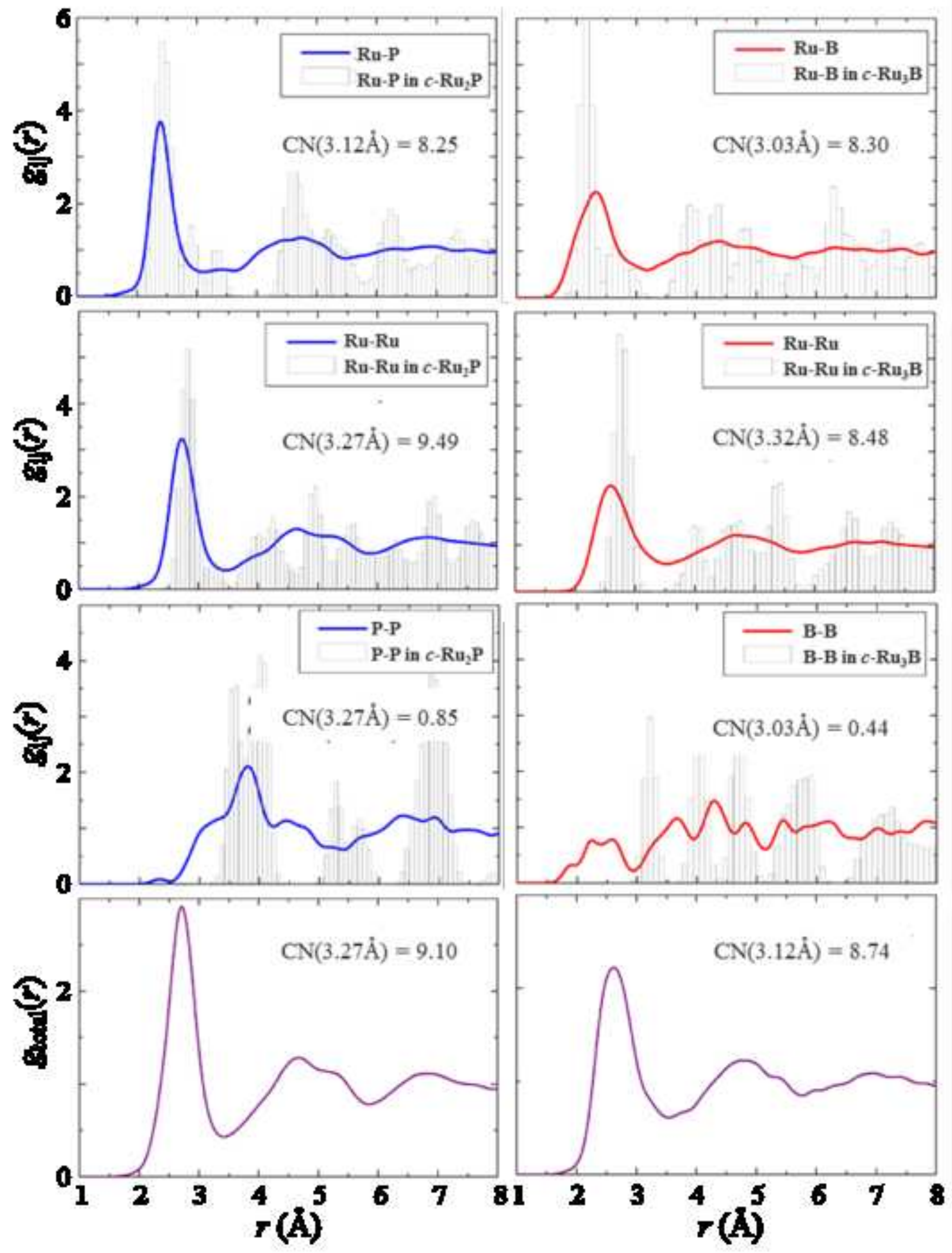


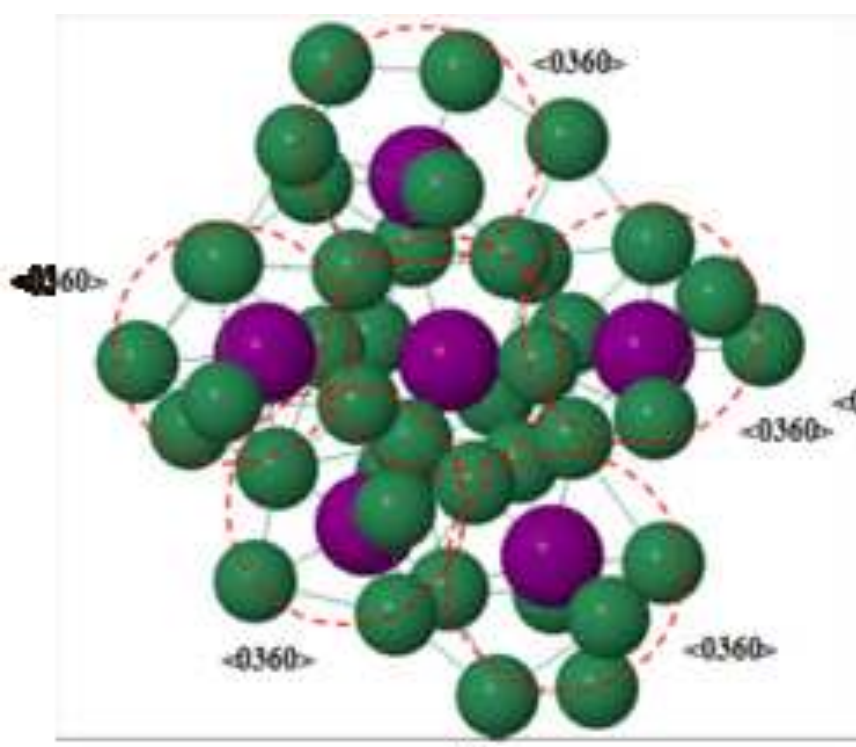

(a)

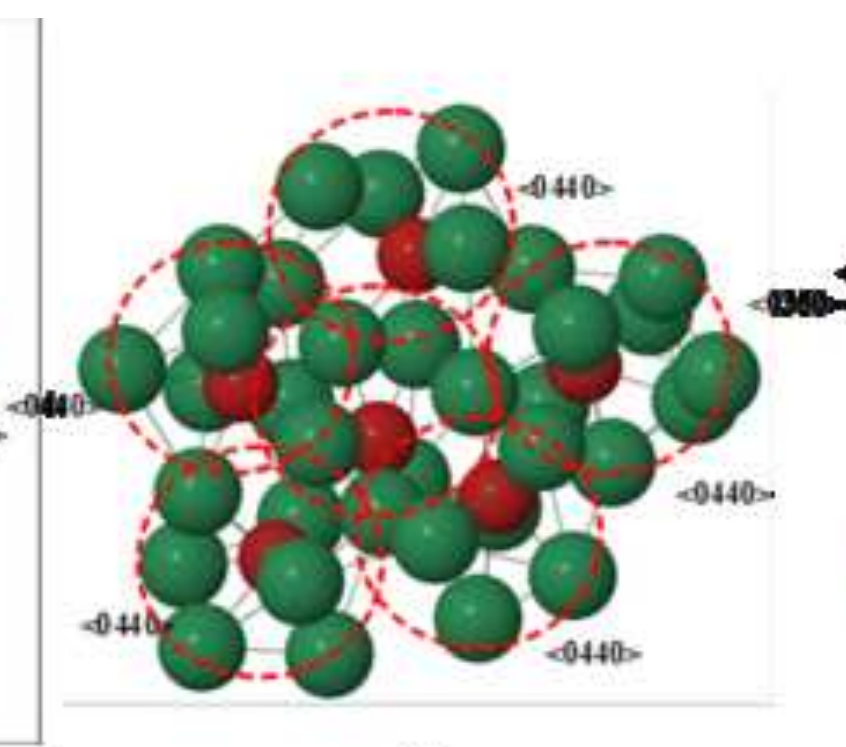

(b)

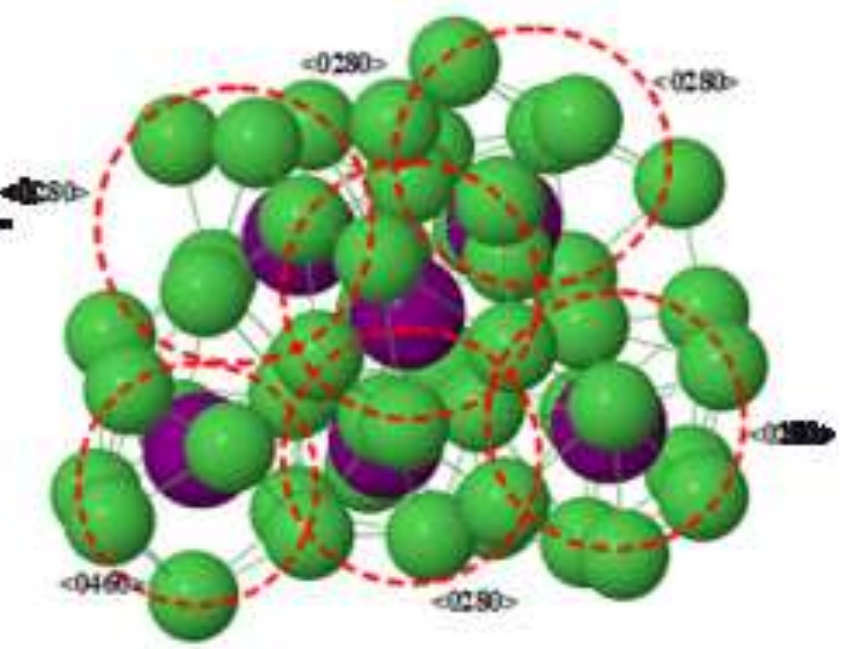

(c) 


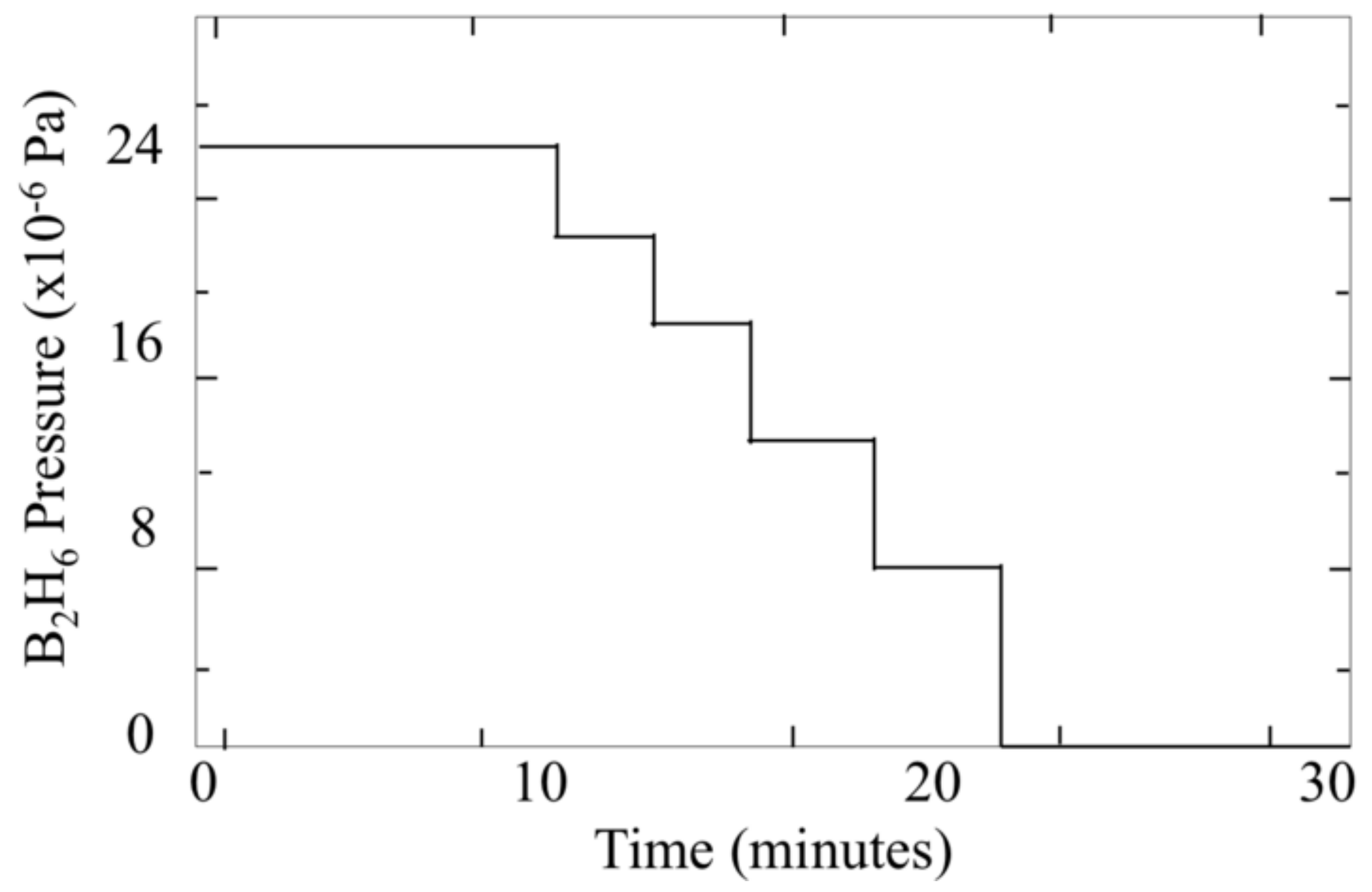




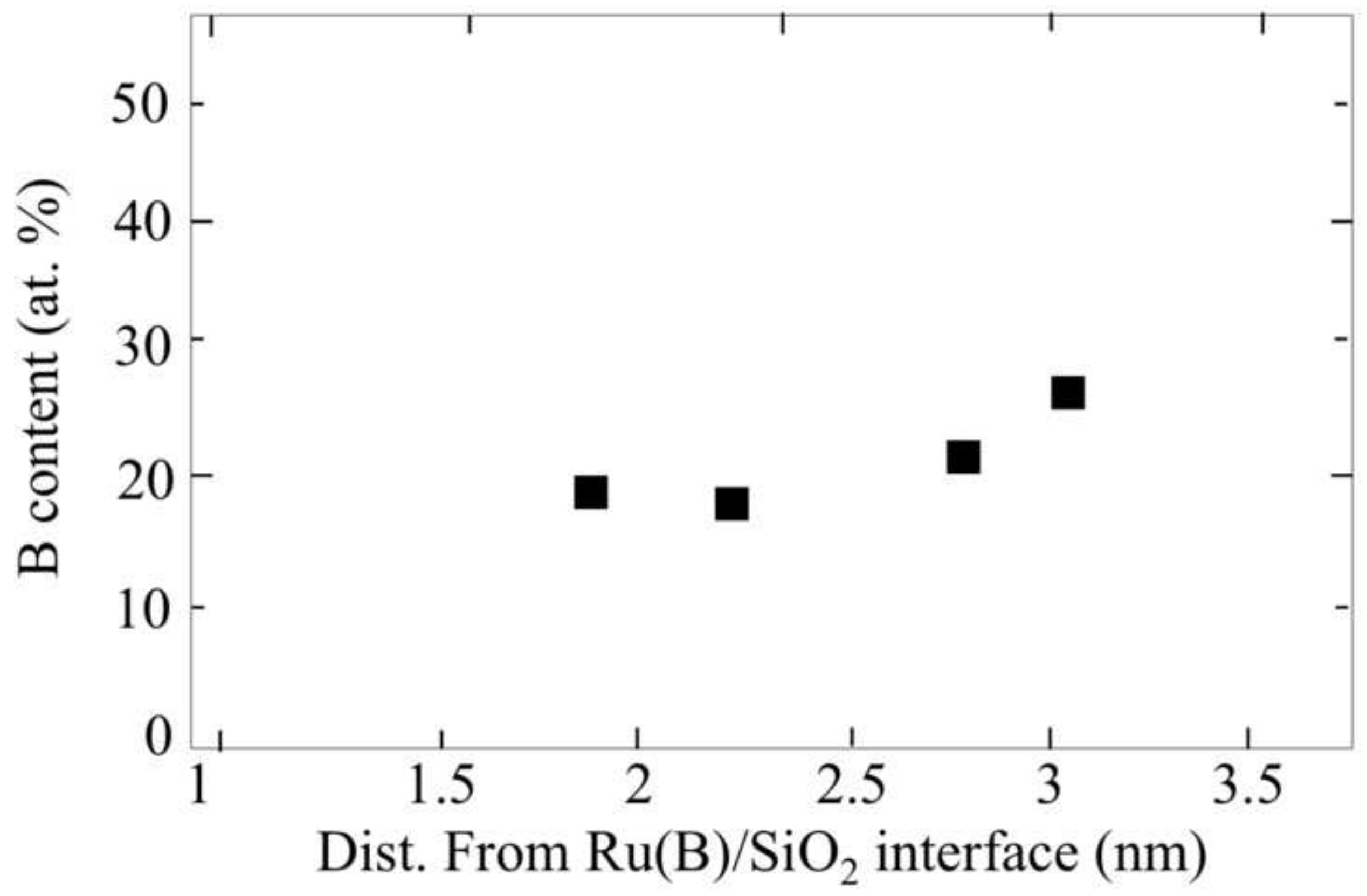




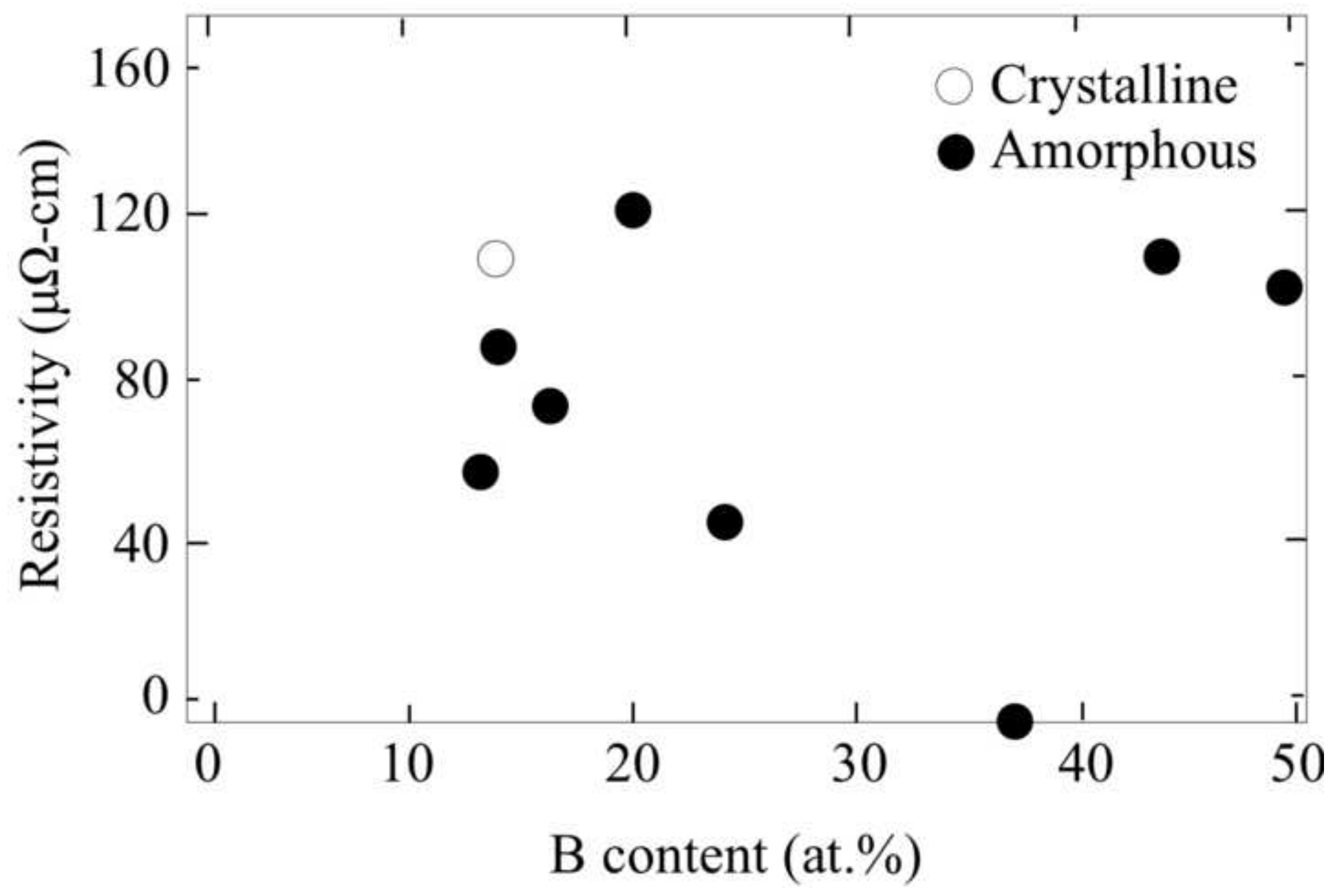




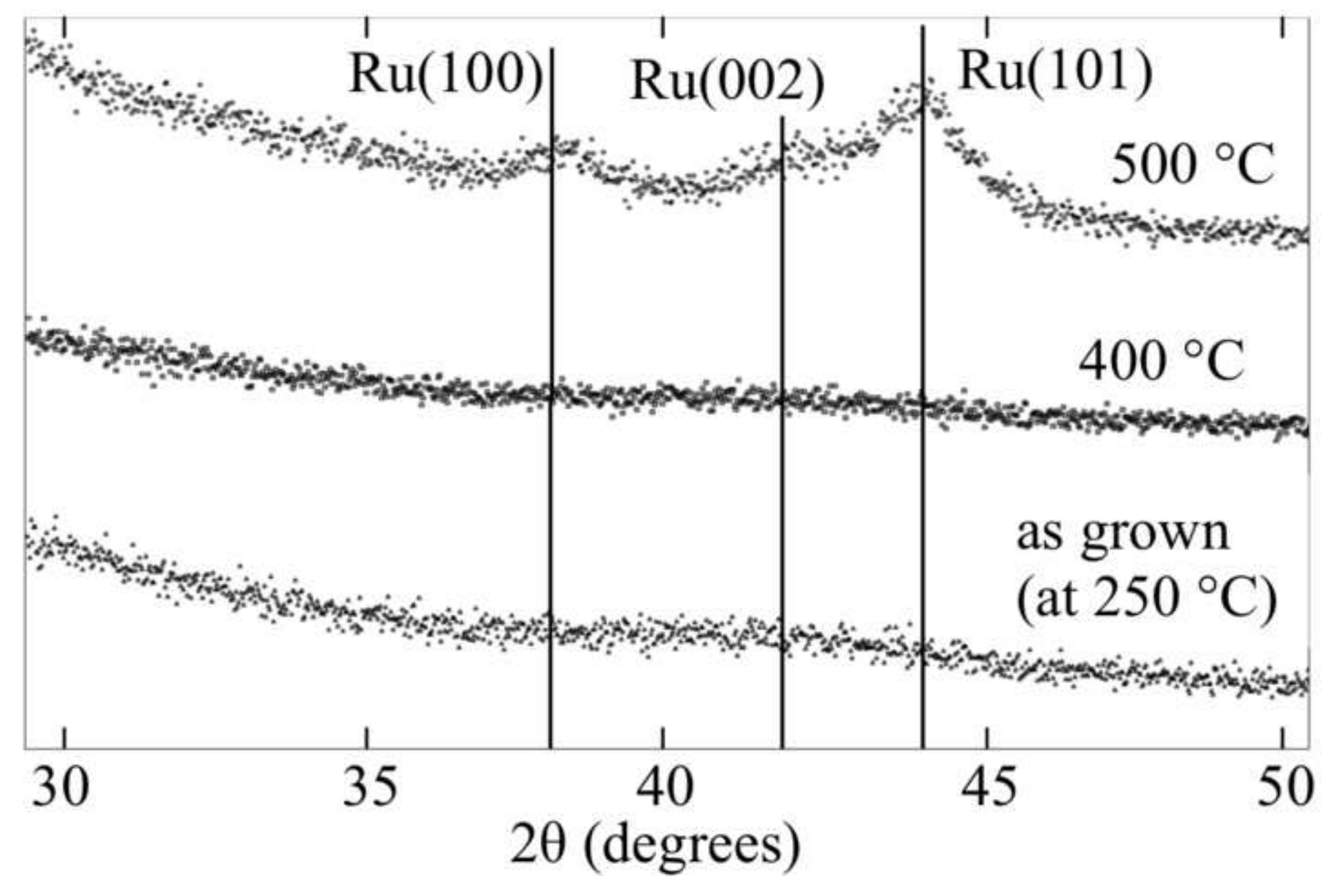




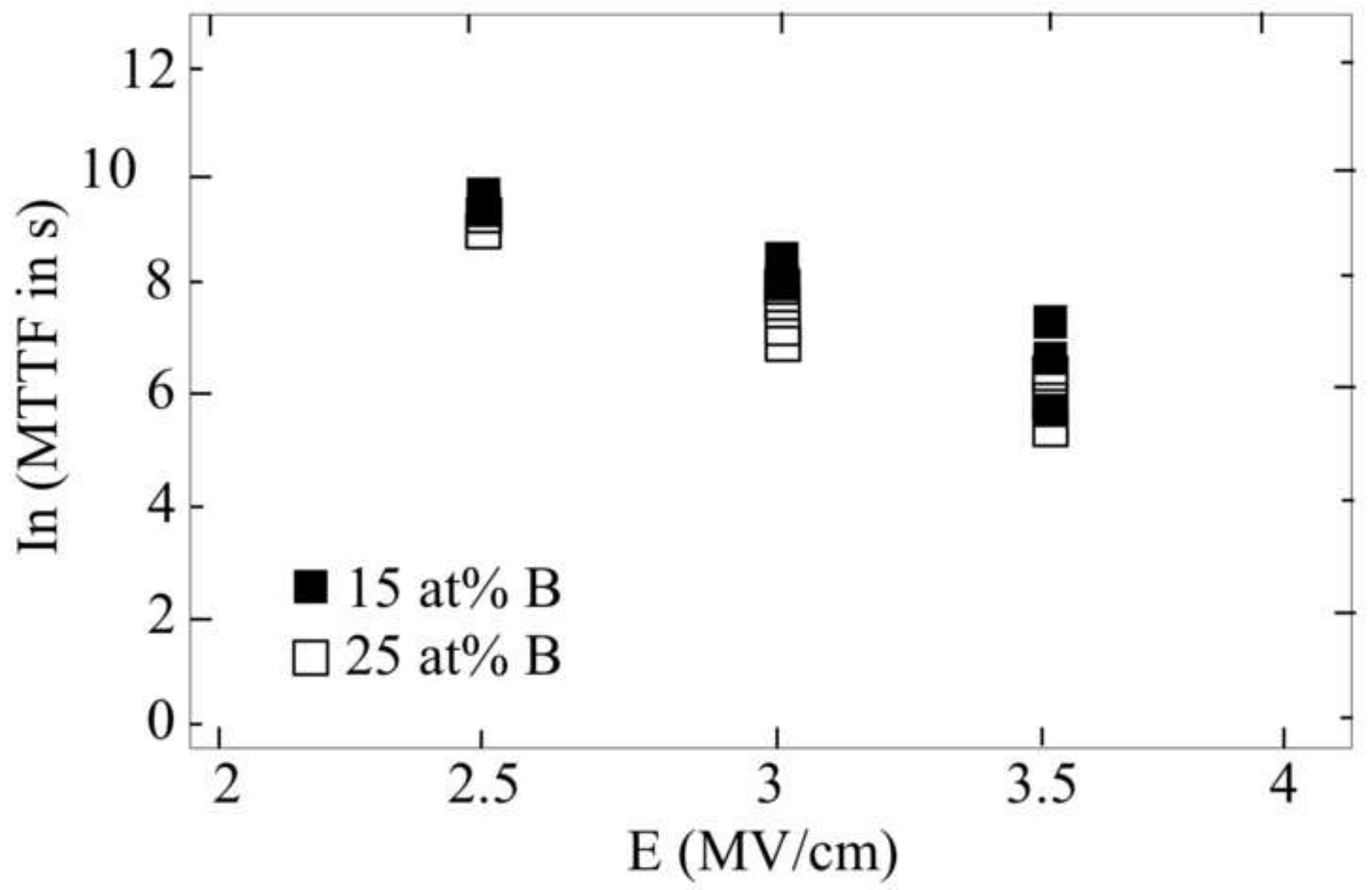




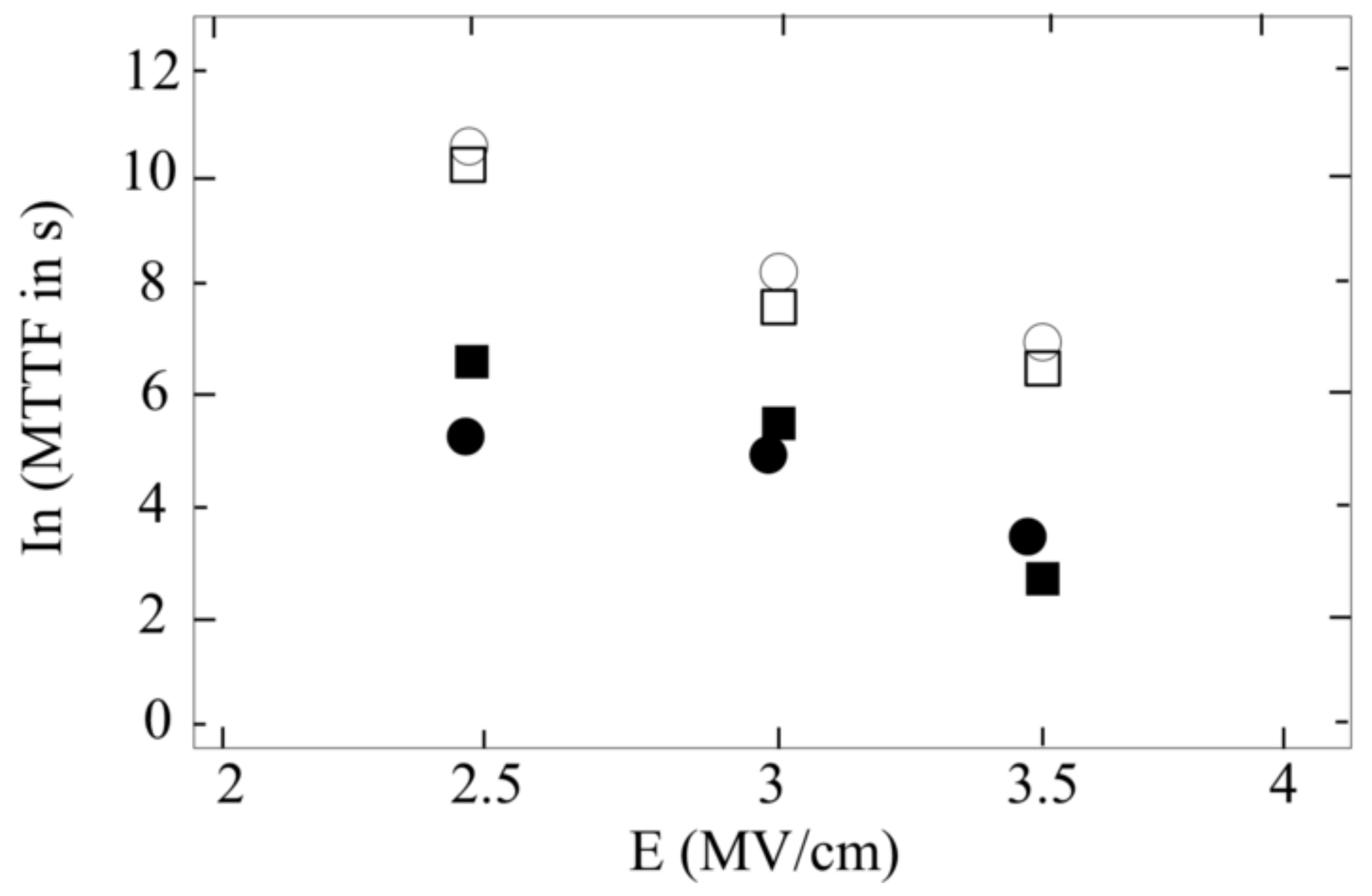

